\title{
Computational modeling suggests impaired interactions between NKX2.5 and GATA4 in individuals carrying a novel pathogenic D16N NKX2.5 mutation
}

\author{
Saidulu Mattapally ${ }^{1, *}$, Mrityunjay Singh ${ }^{2, *}$, Kona Samba Murthy $^{3}$, Shailendra Asthana ${ }^{2}$ \\ and Sanjay K. Banerjee ${ }^{1,2}$ \\ ${ }^{1}$ Division of Medicinal Chemistry and Pharmacology, CSIR-Indian Institute of Chemical Technology, Hyderabad 500007, India \\ ${ }^{2}$ Drug Discovery Research Center (DDRC), Translational Health Science and Technology Institute (THSTI), Faridabad, \\ Haryana 121001, India \\ ${ }^{3}$ Innova Children's Heart Hospital, Tarnaka, Hyderabad 500017, India \\ *These authors have contributed equally to this work \\ Correspondence to: Shailendra Asthana, email: sasthana@thsti.res.in \\ Sanjay K. Banerjee, email: skbanerjee@thsti.res.in \\ Keywords: molecular dynamics simulation; computational modeling; protein-protein interaction; pathogenic mutation; congenital \\ heart disease \\ Received: October 05, $2017 \quad$ Accepted: January 24, $2018 \quad$ Published: February 09, 2018 \\ Copyright: Mattapally et al. This is an open-access article distributed under the terms of the Creative Commons Attribution License \\ 3.0 (CC BY 3.0), which permits unrestricted use, distribution, and reproduction in any medium, provided the original author and \\ source are credited.
}

\section{ABSTRACT}

NKX2.5, a homeobox containing gene, plays an important role in embryonic heart development and associated mutations are linked with various cardiac abnormalities. We sequenced the $N K X 2.5$ gene in 100 congenital heart disease (CHD) patients and 200 controls. Our analysis revealed a total of 7 mutations, 3 in intronic region, 3 in coding region and 1 in $3^{\prime}$ UTR. Of the above mutations, one mutation was found to be associated with tetralogy of fallot (TOF) and two (rs2277923 and a novel mutation, D16N) were strongly associated with VSD. A novel missense mutation, D16N (p-value $=0.009744$ ), located in the tinman (TN) region and associated with ventricular septal defect (VSD), is the most significant findings of this study. Computational analysis revealed that $\mathrm{D} 16 \mathrm{~N}$ mutation is pathogenic in nature. Through the molecular modeling, docking and molecular dynamics simulation studies, we have identified the location of mutant D16N in NKX2.5 and its interaction map with other partners at the atomic level. We found NKX2.5-GATA4 complex is stable, however, in case of mutant we observed significant conformational changes and loss of key polar interactions, which might be a cause of the pathogenic behavior. This study underscores the structural basis of D16N pathogenic mutation in the regulation of $N K X 2.5$ and how this mutation renders the structural-functional divergence that possibly leading towards the diseased state.

\section{INTRODUCTION}

$N K X 2.5$ is one of the transcription factors plays an important role in heart development. It is a cardiac specific homeobox gene and acts as an early marker gene for heart field development. $N K X 2.5$ has been mapped to chromosome 5q31.1 and has two exons [1]. Similar to other members of the nucleotide kinase (NK)-2 class viz. NKX2.1, NKX2.2, NKX2.3, NKX2.4 and NKX2.6, NKX2.5 contains three highly-conserved regions: the homeodomain, the tinman (TN) domain and the NK2 (unique to NK2 class proteins) domain [2]. The homeo-domain is a highly-conserved DNA-binding domain located in the core, while the short TN domain is found at the N-terminal region of most NK-2 proteins [3]. NKX2.5 binds to the consensus sequence of the ANF promoter and interacts with other transcriptional factors such as; GATA4 and TBX5 [4-6]. During fetal heart development, mutation in $N K X 2.5$ 
gene leads to fetal cardiac structural morphogenesis, growth retardation, and embryonic lethality. $N K X 2.5$ knockout mice died from cardiac malformations $[7,8]$. Mutation of $N K X 2.5$ in zebrafish develops embryos with diminutive ventricular and bulbous atrial chambers [9]. Till date, more than 40 heterozygous $N K X 2.5$ germline mutations associated with CHD patients were reported [10-13]. However, the mechanisms by which mutations cause cardiac defects remain largely unknown.

CHD is a multifactorial disease in which genetic and environmental factors play an important role to develop the disease. Genetic events such as mutations and chromosomal aberrations are responsible for CHD $[14,15]$. Several studies reported that $N K X 2.5$ gene mutations cause different types of CHDs like atrial septal defect (ASD), ventricular septal defects (VSD), tetralogy of fallot (TOF) and single ventricle (SV). Recent studies reported that the prevalence of $N K X 2.5$ mutations is about $1-4 \%$ in sporadic patients with ASD [11]. VSD occurs in approximately $50 \%$ of all children with CHD, and accounts for 14 to $16 \%$ of the defects that require an invasive procedure within the first year of life $[16,17]$. VSD can occur alone or with other cardiac anomalies, such as ASD, down-syndrome, or TOF. Compared to other countries, the prevalence of CHD in India is considerably higher [18]. However, very few studies on atomic level at structural basis have been conducted to find the mutational causes of CHD.

We identified a novel D16N mutation in $N K X 2.5$ by screening 100 South Indian CHD patients. We are elucidating the molecular basis as how this mutation becomes pathogenic and cause CHD through proteinprotein interaction study. The structural localization of D16N mutation and their atomic level resolution showed as how this mutation alters the architecture of functional protein-protein and/or protein-DNA interactions. Additionally, we also tried to explore the structural understanding of disease associated reported mutations. It is known that GATA4-NKX2.5 partnership may represent a paradigm for transcription factor interaction during organogenesis [5]; however, the mode of interaction has not been reported yet. The protein-protein interaction between $N K X 2.5$ and GATA4 provides the structural insights as how D16N mutation perturbed the structuralfunctional relationship, which might be responsible for pathogenicity.

\section{RESULTS}

\section{Clinical evaluation and mutations in NKX2.5 gene}

We collected sample from a total of $100 \mathrm{CHD}$ patients. Sample selection was based on two criteria, first, was CHD types (ASD, VSD, TOF, and SV) and second, was demographics, i.e. selection of the study population based on geographical location, speaking the Dravidian language (Dravidians) and living in southern India. The percentage of CHD patients belong to different categories are as follows; ASD: 33\%, VSD: $32 \%$, TOF: 32\%, SV: $3 \%$. Age of all CHD patients is ranging from 0.35 to 10.79 years. However, maximum number of CHD patients used for this study was $<5$ years [19]. Two hundred individuals having no CHD or any family history of CHD or heart disease were included in this study as control. The study conforms to the principles outlined in the Declaration of Helsinki and was approved by the research advisory committee and institutional ethical committee of Innova Children's Heart Hospital, Hyderabad.

Our analysis revealed a total of 7 mutations, out of which 4 mutations were in intronic regions, 2 mutations were in exonic region (one missense and one synonymous mutation) and 1 mutation was in 3' UTR (Table 1). We classified the samples based on phenotype: ASD, VSD, TOF, and SV and removed one marker, which were not following Hardy-Weinberg equilibrium in ethnically matched control samples. This marker was rs703752 $($ HWE p-value $=0.003877)($ Supplementary Figure 1$)$. In-silico analysis showed that one missense mutation was conserved and predicted to be pathogenic (Figure 1A and 1B). Further, we performed Chi-square analysis for finding the statistical significance. In TOF, rs2277923 (p-value = 0.0002528 ) was associated with the disease. In case of VSD, 2 mutations have shown strong association. They are rs2277923 (p-value $=2.142 \mathrm{e}-07$ ) and novel D16N (p-value $=0.009744)($ http://www.ashg.org/2014meeting/ abstracts/fulltext/f140121487.htm; dbSNP reference numbers: NM_001166175.1:c.46G $>$ A, NM_001166176.1: c.46G $>$ A, NM_004387.3: c.46G $>$ A, XM_017009071.1: c. $46 \mathrm{G}>\mathrm{A}$ ) (Figure 2, Table 2).

\section{In-silico analysis of missense mutation}

In the coding region, we found one missense mutations and one synonymous mutation in CHD patients. These two mutations are D16N and E63E, respectively. Multiple alignments of $N K X 2.5$ amino acid sequences from human, cattle, monkey, pig, dog, rat and mouse found that D16 is conserved during evolution (Figure 1B). To know the functional significance of missense mutations, we performed bioinformatics analysis of identified missense mutation using PMut (http://mmb. irbbarcelona.org/PMut/), SIFT (http://sift.bii.astar.edu. sg/), PROVEAN (http://provean.jcvi.org/seq submit. php), PANTHER (http://www.pantherdb.org/tools/ csnpScoreForm.jsp), PHD-SNP (http://snps.biofold.org/ phd-snp/phd-snp.html), SNAP (https:/www.rostlab.org/ services/snap/) and PredictSNP (https://loschmidt.chemi. muni.cz/predictsnp1/). These tools predict whether an amino acid substitution or indel has an impact on the biological function of a protein or not. 
Table 1: List of all $N K X 2.5$ mutations identified in the present study

\begin{tabular}{|c|c|c|c|c|c|c|c|c|}
\hline S. No. & dbSNP-ID & $\begin{array}{c}\text { Physical position } \\
\text { (hg19) }\end{array}$ & $\begin{array}{c}\text { Nucleotide } \\
\text { variation }\end{array}$ & $\begin{array}{c}\text { Mutation } \\
\text { type }\end{array}$ & AA change & Observe hetero. & Expected hetero. & P-value: HWE \\
\hline 1 & rs 703752 & 172659511 & $\mathrm{G}>\mathrm{T}$ & 3'UTR & - & 0.03 & 0.04875 & 0.003877 \\
\hline 2 & Novel-Intronic & 172659740 & $\mathrm{C}>\mathrm{G}$ & Intronic & - & 0.155 & 0.143 & 0.612 \\
\hline 3 & CM086533 & 172661873 & $\mathrm{G}>\mathrm{A}$ & Intronic & - & 0.17 & 0.1556 & 0.3696 \\
\hline 4 & rs2277923 & 172662024 & $\mathrm{G}>\mathrm{A}$ & Synonymous & E63E & 0 & 0 & 1 \\
\hline 5 & Novel-Exonic & 172662041 & $\mathrm{G}>\mathrm{A}$ & Missense & $\mathrm{D} 16 \mathrm{~N}$ & 0 & 0 & 1 \\
\hline 6 & rs3729937 & 172662129 & $\mathrm{C}>\mathrm{T}$ & Intronic & - & 0.035 & 0.03439 & 1 \\
\hline 7 & rs77083308 & 172662192 & $\mathrm{G}>\mathrm{A}$ & Intronic & - & 0.09 & 0.08595 & 1 \\
\hline
\end{tabular}

The outcome of PMut, PROVEAN, SIFT, PANTHER, PHD-SNP, SNAP and PredictSNP strongly indicate that the D16N mutation is pathogenic (Table 3 ). Hence to explore the structural mechanistic aspect, how $\mathrm{D} 16 \mathrm{~N}$ mutation leads to the disease state, we performed the extensive computational structural dynamics study.

\section{Structural localization of D16N in TN domain of NKX2.5 and its interaction map}

\section{Molecular modeling}

Structural localization of mutation is essential to relate its effects on protein functional phenotype. We carried out protein-DNA interaction, protein-protein interaction, and molecular dynamic simulation studies to know how D16N@NKX2.5 mutation renders the protein resistant and paving its pathway towards the diseased state. We believe either it perturbs the interaction with ANF-242 promoter DNA or disturbing the association with other transcription factor viz. GATA4, TBX5. To answer this question, it is fundamental to determine, complete structure of $N K X 2.5$ specially $\mathrm{N}$-term region and interaction pattern of the residue D16 with substrates like ANF-242 promoter (DNA), GATA4 and TBX5. We performed our modeling studies in a systematic way as we constructed different sets of model complex systems: NKX2.5 (apo), NKX2.5-DNA, NKX2.5-GATA4, NKX2.5-TBX5, NKX2.5-DNA-GATA4, and NKX2.5DNA-TBX5. To explore the effect of D16N we first evaluated the vicinity of D16 residue from the DNA and other substrate. Since the crystal structure of complete sequence of $N K X 2.5$ and GATA4 has not been reported so far, except, the homeodomain (HD) of $N K X 2.5$ [6, 20] and Zn-finger domain of GATA4 (PDB ID: 2M9W). Therefore, molecular modeling was carried out to model DNA (ANF-promoter), NKX2.5 and GATA4 complex assembly. A schematic diagram of $N K X 2.5$ and GATA4 proteins depicting the structural domains and the location of the mutation D16N@NKX2.5 detected in present study is delineated in Figure 1C.

\section{Modeling of the DNA (ANF-242 promoter)}

Human ANF promoter is the key, direct, and downstream target of $N K X 2.5$, which involve in cardiac development. Each transcription factor binds to its own specific binding motif in DNA, and for each transcription factor, ANF promoter has many several me-too binding motifs $[5,6]$. Therefore, it is interesting to identify the most likely binding motif.

Through JASPER tool [21] we predicted the binding site of transcription factor in promoter-DNA with the help of motif matrix model. We found 16 putative binding sites in ANF promoter region (Supplementary Table 1). Recently, Pradhan et. al. highlighted that $N K X 2.5$ binds preferentially in human ANF promoter DNA at "-242AAGTG” motif with TT at its 5'terminal (TTAAGTG). So, we search motif “AAGTG” in identified 16 NKE motifs. Out of 16 , we found two motifs at position -242 and -80 matched with AAGTG motif (Figure 1D and S2B and, Supplementary Table 1). It is reported that ANF80 site has weak binding affinity with $N K X 2.5$ than ANF242 site [6]. Additionally, the ANF-242 binding region is also known for binding of other transcription factor like GATA4. It is well documented that interaction of GATA4 necessary for activation of $N K X 2.5$ [5]. Due to the abovementioned reasons, the ANF-242 was considered for model building steps.

\section{Modeling of $\mathrm{NKX} 2.5$}

In absence of crystal data of $N K X 2.5$ (except HD region) the dis-orderedness analysis followed [22] by globularity prediction [23] was performed to get the clues that how much $N K X 2.5$ is structured, intrinsically disordered protein (IDP) and complete disordered. It is a known fact that TFs are undergone disorder-to-order transitions upon binding to other substrates and/or partners protein [24, 25]. Similarly, several folded proteins regulate order-to-disorder transition to mediate their biological function/s [25-27]. Therefore, the information of binding regions (BRs), IDPs and completely disordered regions were used for the comparative model building of $\mathrm{N}$-term region of $N K X 2.5$. In the multi-step approach delineated as a scheme1: flowchart 

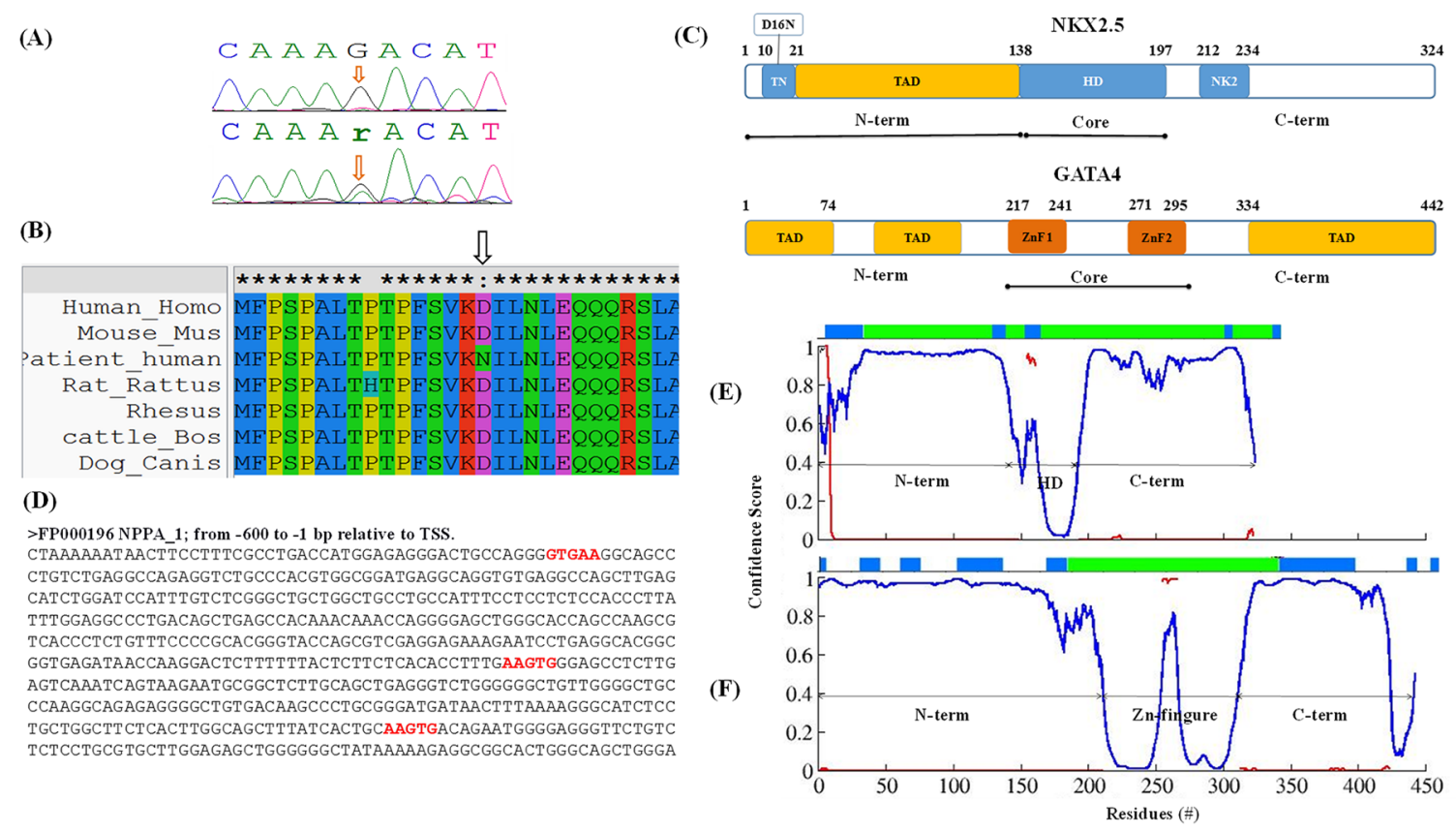

Figure 1: (A) DNA sequencing electropherogram showing wild type (upper panel) and heterozygous (G $\rightarrow$ A) mutation (lower panel) in $N K X 2.5$ coding region (arrows), mutation D16N, (B) Multiple amino acid sequence alignment of different species shows the conservation of the mutated amino acid residue (amino acid marked) across species. (C) Schematic diagram of NKX2.5 and GATA4. NKX2.5: structure/ architecture depicting the location of a novel mutation D16N; N-term, amino-terminal region; TN, tin-man domain; HD, homeo-domain; NK2, nucleotide kinase domain-2; C-term, carboxy-terminal region. GATA4: TAD, transcription activation domain; two Zn finger motifs, ZnF1 (BR2) and ZnF2 (BR1). Three regions, N-term (amino-terminal region); core and C-term (carboxy-terminal region) are mentioned. The molecular modeling studies were conducted on N-term + core in case of NKX2.5, and only core in GATA4, is highlighted by horizontal black lines. (D) Sequence of Atrial natriuretic factor (ANF) promoter region from $-600 \mathrm{bp}$ to $-1 \mathrm{bp}$. The motifs shown in red color are potential binding motifs for human $N K X 2.5$ in human ANF promoter, identified from literature. In panels (E and F) Structural disorder and globularity predictions: the disorder regions (confidence score higher than 0.5 ) are shown by blue lines and protein binding region shown by red lines. Confidence score shown in Y-axis, and residues in X-axis. Globularity prediction is shown as horizontal bar at the top, disordered region (in blue), ordered region (in green) and the gaps represent coil regions, (E) NKX2.5 and (F) GATA4.

\section{Atrial septal defect}

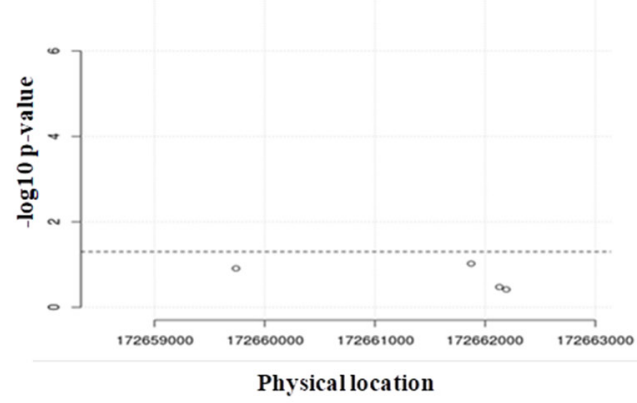

Tetralogy of fallot

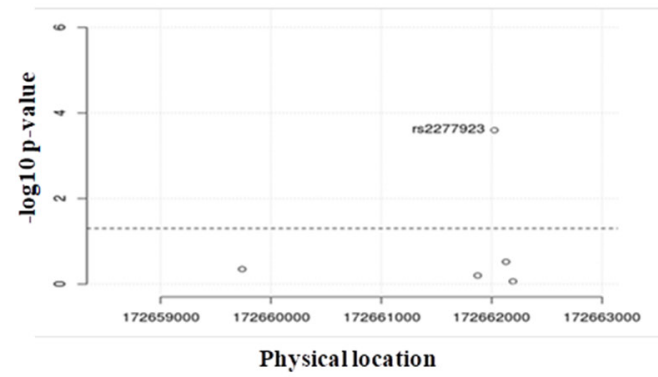

Ventricular septal defect

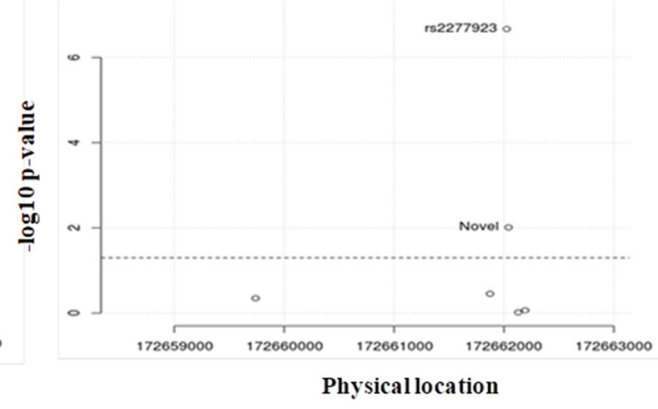

Single ventricle

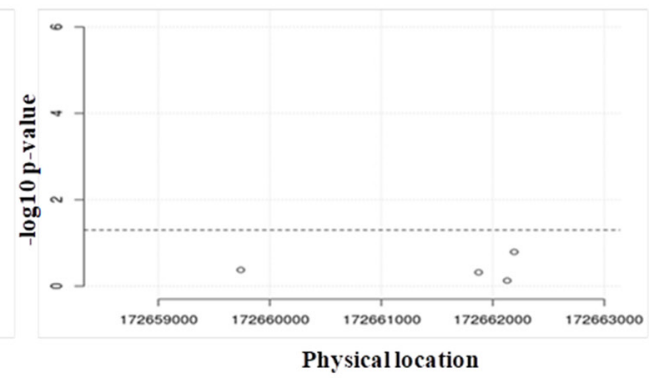

Figure 2: Chi-square P-value of variations found in NKX2.5 of all types of CHD. (A) atrial septal defect (ASD), (B) ventricular septal defect (VSD), (C) tetralogy of fallot (TOF) and (D) single ventricle (SV). 
Table 2: Significance test for $N K X 2.5$ mutations

\begin{tabular}{|c|c|c|c|c|c|c|c|c|c|c|}
\hline 苞 & CHR & db-SNP-ID & $\begin{array}{c}\text { Physicalposition } \\
\text { (hg19) }\end{array}$ & 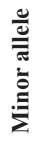 & $\begin{array}{l}\text { Frequency of } \\
\text { minor allele in } \\
\text { cases }\end{array}$ & $\begin{array}{l}\text { Frequency of minor } \\
\text { allele in control }\end{array}$ & 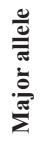 & Chi-sq. value & P-value & OR \\
\hline \multirow{6}{*}{ के } & 5 & Novel-Intronic & 172659740 & A & 0.01923 & 0.0775 & G & 2.375 & 0.1233 & 0.2334 \\
\hline & 5 & CM086533 & 172661873 & A & 0.01923 & 0.085 & G & 2.786 & $9.51 \mathrm{E}-02$ & 0.2111 \\
\hline & 5 & rs 2277923 & 172662024 & A & 0 & 0 & $\mathrm{G}$ & NA & NA & NA \\
\hline & 5 & Novel-Exonic & 172662041 & $\mathrm{G}$ & 0 & 0 & $\mathrm{C}$ & NA & NA & NA \\
\hline & 5 & rs3729937 & 172662129 & $\mathrm{~T}$ & 0 & 0.0175 & $\mathrm{C}$ & $9.24 \mathrm{E}-01$ & 0.3363 & 0 \\
\hline & 5 & rs77083308 & 172662192 & A & 0.01923 & 0.045 & $\mathrm{G}$ & 0.7589 & 0.3837 & 0.4161 \\
\hline \multirow{6}{*}{$\stackrel{0}{>}$} & 5 & Novel-Intronic & 172659740 & A & 0.05 & 0.0775 & G & 0.5764 & 0.4477 & 0.6265 \\
\hline & 5 & CM086533 & 172661873 & A & 0.05 & 0.085 & $\mathrm{G}$ & 0.8641 & 0.3526 & 0.5666 \\
\hline & 5 & $r s 2277923$ & 172662024 & $A$ & 0.06667 & 0 & $G$ & 26.9 & $2.14 E-07$ & $N A$ \\
\hline & 5 & Novel-Exonic & 172662041 & $G$ & 0.01667 & 0 & $C$ & 6.681 & 0.00974 & $N A$ \\
\hline & 5 & rs3729937 & 172662129 & $\mathrm{~T}$ & 0.01667 & 0.0175 & $\mathrm{C}$ & 0.00212 & 0.9633 & 0.9516 \\
\hline & 5 & rs 77083308 & 172662192 & A & 0.05 & 0.045 & $\mathrm{G}$ & 0.02994 & 0.8626 & 1.117 \\
\hline \multirow{6}{*}{$\stackrel{\sqrt[T]{O}}{O}$} & 5 & Novel-Intronic & 172659740 & A & 0.05 & 0.0775 & G & 0.5764 & 0.4477 & 0.6265 \\
\hline & 5 & CM086533 & 172661873 & A & 0.06667 & 0.085 & $\mathrm{G}$ & 0.2314 & 0.6305 & 0.7689 \\
\hline & 5 & $r s 2277923$ & 172662024 & $A$ & 0.03333 & 0 & $G$ & 13.39 & 0.00025 & $N A$ \\
\hline & 5 & Novel-Exonic & 172662041 & $\mathrm{G}$ & 0 & 0 & $\mathrm{C}$ & NA & NA & NA \\
\hline & 5 & rs3729937 & 172662129 & $\mathrm{~T}$ & 0 & 0.0175 & $\mathrm{C}$ & 1.066 & 0.3018 & 0 \\
\hline & 5 & rs 77083308 & 172662192 & A & 0.05 & 0.045 & $\mathrm{G}$ & 0.02994 & 0.8626 & 1.117 \\
\hline \multirow{6}{*}{$\pi$} & 5 & Novel-Intronic & 172659740 & A & 0.1667 & 0.0775 & $\mathrm{G}$ & 0.6473 & 0.4211 & 2.381 \\
\hline & 5 & CM086533 & 172661873 & A & 0.1667 & 0.085 & $\mathrm{G}$ & 0.5005 & 0.4793 & 2.153 \\
\hline & 5 & $r s 2277923$ & 172662024 & $A$ & 0.1667 & 0 & $G$ & 66.83 & $2.96 E-16$ & $N A$ \\
\hline & 5 & Novel-Exonic & 172662041 & G & 0 & 0 & $\mathrm{C}$ & NA & NA & NA \\
\hline & 5 & rs3729937 & 172662129 & $\mathrm{~T}$ & 0 & 0.0175 & $\mathrm{C}$ & 0.1068 & 0.7438 & 0 \\
\hline & 5 & rs 77083308 & 172662192 & $\mathrm{~A}$ & 0.1667 & 0.045 & G & 1.962 & 0.1613 & 4.244 \\
\hline
\end{tabular}

Table 3: Prediction of functional significance of the D16N NKX2.5 mutation by using multiple computational programs

\begin{tabular}{lccc}
\hline \multirow{2}{*}{ Name of software } & \multicolumn{2}{c}{ D16N } & \multirow{2}{*}{ Cut-off } \\
\cline { 2 - 4 } & Prediction & Score & 0.5 \\
PMut & Pathological & $0.72(86 \%)$ & 0.05 \\
SIFT & Pathological & 0 & -2.5 \\
PROVEAN & Pathological & -3.75 & 450 \\
PANTHER & Pathological & 1037 & 0.5 \\
PHD-SNP & Pathological & 0.674 & $0.50 \%$ \\
PredictSNP & Pathological & $0.72 \%$ & 0.5 \\
PolyPhen-2 & Benign & 0.029 & \\
\hline
\end{tabular}

of molecular modeling pipeline (Figure 3), the quality assessment of models (fragments optimization and linking of fragments, and loop optimization) was verified through multi-round analysis via ramachandran plot [28], SASA
[29], RMSD, ERRAT [30], ProSA_Zscore [31], Dope_ Score [32] and secondary structure analysis. Final models were optimized through molecular dynamics simulations (see supplementary data for more details). 
Molecular modeling was performed separately for $\mathrm{N}$-term and HD domains of $N K X 2.5$ through different approaches and then validated separate domains were concatenated to get the desired final models (Scheme 1). The concatenation is only continuing when it fulfilled the validation and optimization cut-offs and each step was verified through model analysis steps followed by the energy minimization and simulation through molecular dynamics (Scheme1, Figure 3). The results show that the final model of $N K X 2.5$ is stable during the dynamics from 20 to $100 \mathrm{~ns}$ (Figure 4C). The closest to the average structure was extracted from last $80 \mathrm{~ns}$ of the MD simulation and a Ramachandran plot was performed for model accuracy check (Supplementary Figure 3). The model was further evaluated by Prosa Z-score, which is $-4.57(4 \mathrm{SOH}),-4.54$ (model HD@NKX2.5), -4.28 (model N-term@NKX2.5) and -4.0 (model complete NKX2.5 N-term+ HD part) (Supplementary Figure 4). The ERRAT values are 98.0 (4SOH), 94.8 (3RKQ) and 91.7 and (model: N-term+HD) (Supplementary Figure
5). These values are supported well with Ramachandran scores, which are 92.0\% (4S0H), 97.8\% (model HD), 86.7\% (model N-term) and 89.7\% (model: N-term+HD) (Supplementary Figure 3). The same steps were carried out for GATA4. See supplementary data for more details.

\section{Identification of disorder, binding and globular regions}

The amino acid residue from 1 to 30 and 315 to 324 are moderately disordered (confidence score 0.6-0.8), residue 30 to 130 and 195 to 315 are highly disordered (confidence score $>0.8$ ), residue 130 to 140 are partial disordered (confidence score $\sim 0.6$ ) and residue 140 to 195 are structured (confidence score $<0.5$ ) (Figure 1E). DISOPRED used DISOPRED2 dynamic disorder prediction method provides accurate estimation of disorder prediction of around 93.1\% [33].

The protein binding regions in intrinsic disorder proteins (IDPs) may undergo disorder-to-order transition

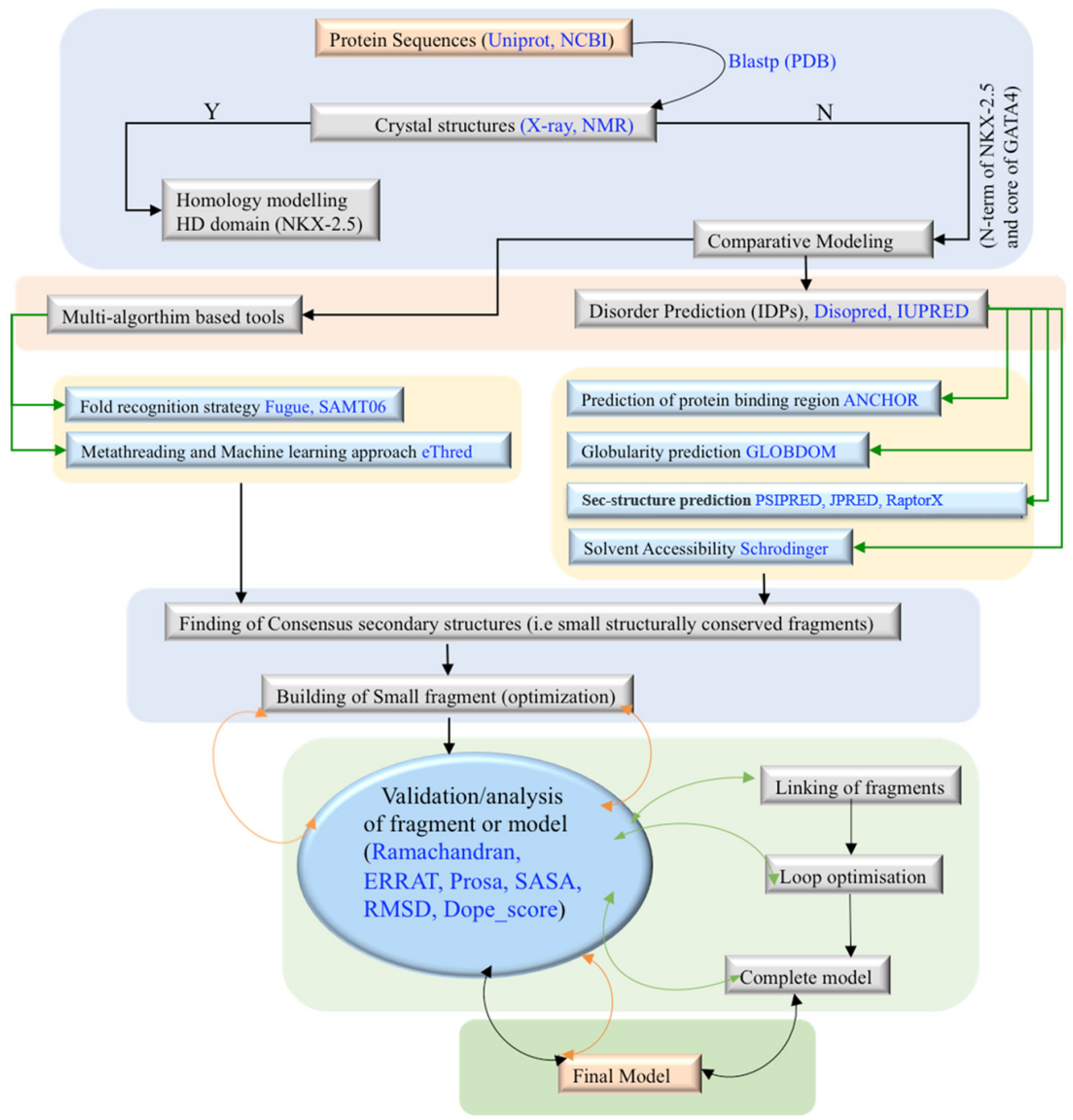

Figure 3: Scheme1: Flowchart of molecular modeling pipeline. 
during protein binding state and acquired specific secondary structure to execute their biological function [34]. Hence, we also checked the protein binding regions through ANCHOR [34] program. The prediction of binding regions is based on estimating the energy content in free and bound states, and identifying segments that are potentially sensitive to these changes. Identification of these segments is based on motif search algorithm.

(A)

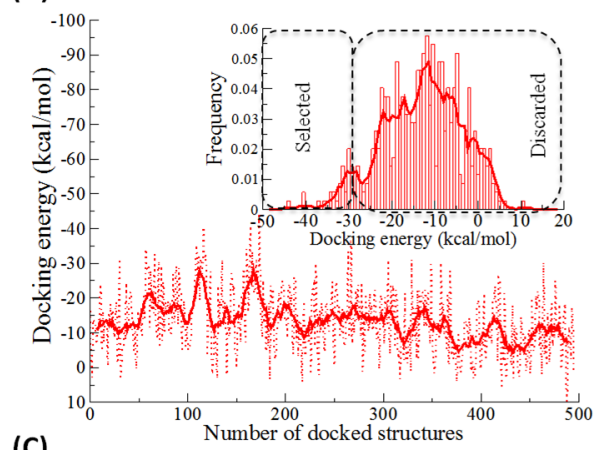

(C)
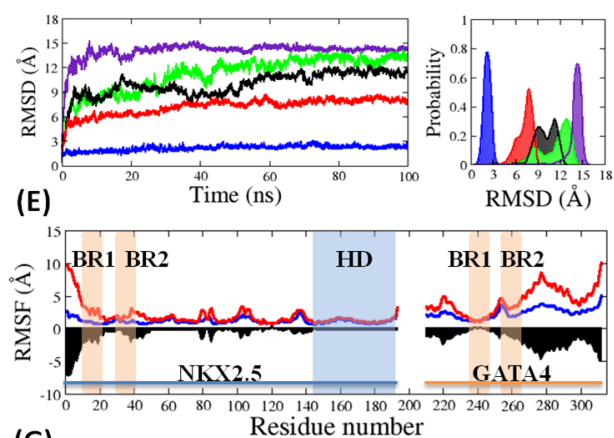

(G)
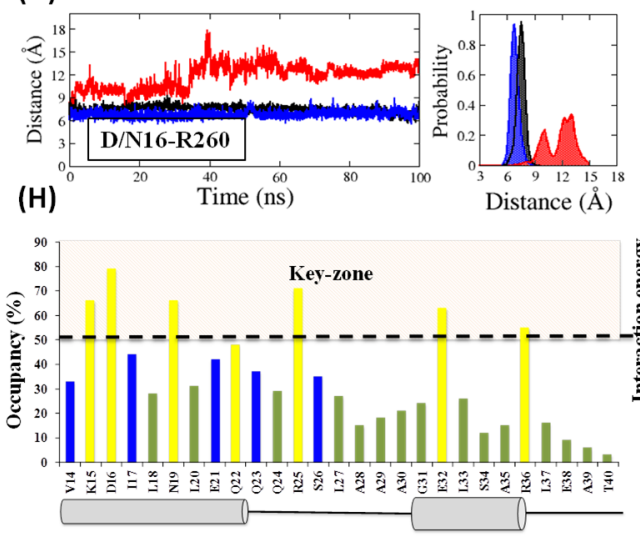

It was suggested that interaction with certain proteins or protein families are mediated through specific linear motifs. A growing number of such linear motifs are now being categorized through ELM server [35]. The anchor tool identified the three most prominent protein binding regions in $N K X 2.5$ : TN domain (at $\mathrm{N}$-term), HD region, and C-term (Figure 1E). The $\alpha 1$ helix (residue 146-159) HD region of $N K X 2.5$ interacts with $T B X 5$ [20] while

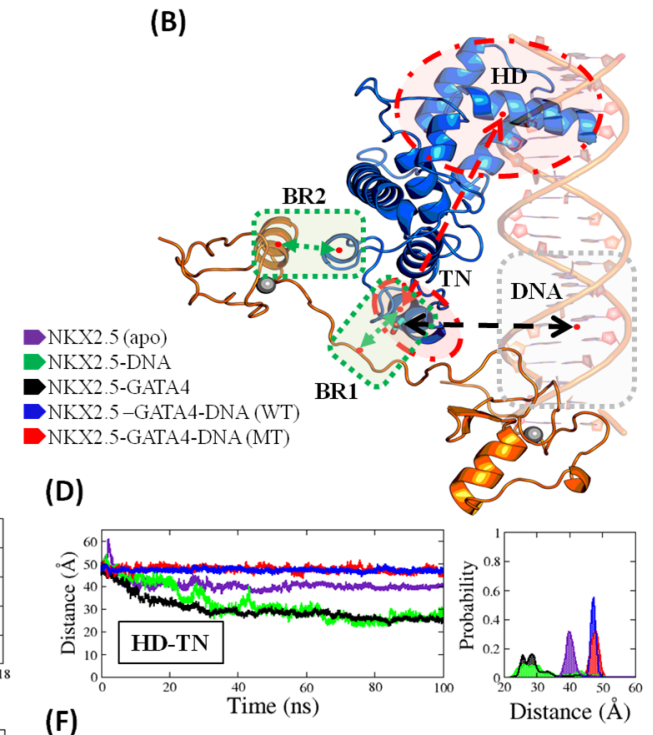

(F)

(B)
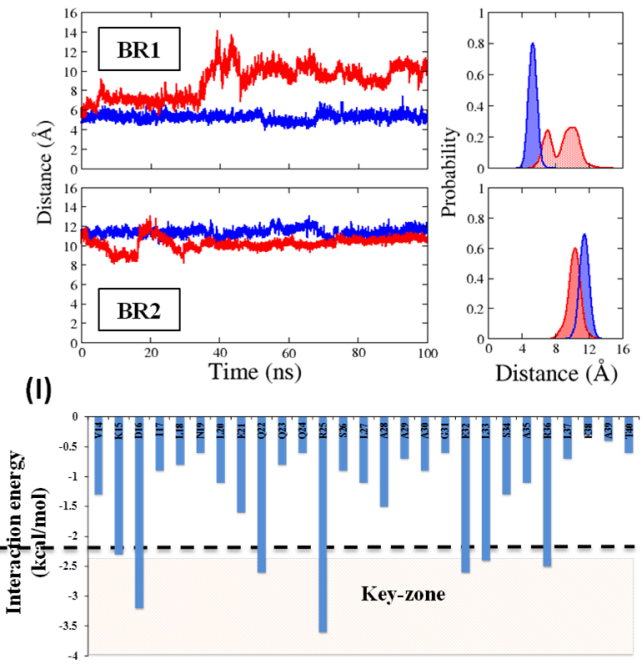

Figure 4: Molecular dynamics simulation analysis: (A) Selection of best-docked pose of NKX2.5 and GATA4 (complexes) from Protein-protein interface through PP-Docking. Histogram differentiates between zone of selected conformers and discarded conformers of complex. (B) Cartoon representation of NKX2.5 (D16)-DNA-GATA4 (WT) complex with highlighted domain movement (HD-TN, distance between center of mass of HD and TN domain of NKX2.5) in red colour, distance between TN@NKX2.5 and DNA in black colour and distance between NKX2.5 and GATA4 at binding region (BR1 and BR2) in green colour. In panels (C to G) the colour code for the MD systems are: $N K X 2.5$ (apo, in violet), $N K X 2.5$ - DNA (in green), $N K X 2.5$-GATA4 (in black), NKX2.5-DNA-GATA4 (WT, in blue) and NKX2.5-DNA-GATA4 (MT, in red). (C) The Root mean square deviation (RMSD, all backbone atoms) in coordinates as a function of the molecular dynamics simulation time. (D) The Domain movement between HD domain and TN domain throughout simulation. (E) RMSF value of Ca atoms comparing WT complex and MT complex simulations. (F) Distance analysis at BR1 and BR2 in WT and MT (G) Distance comparison between D/N16 and R260 in WT and MT complex system throughout simulation. (H) Occupancy of hydrogen bonds at BR1 site. The secondary structure helixes are shown by tubes and coils. (I) Residue wise interaction energy analysis (in kcal/mol). 
C-term of $N K X 2.5$ interacts with C-term region of GATA4 [5]. Our models corroborate well with these finding, which confirmed that Binding Region (BR) prediction for model building was selected with high accuracy. We found in NKX2.5-GATA4 protein-protein interaction study that, the protein binding zone at region TN domain@NKX2.5 interact with protein binding zone in region ZnF@GATA4.

Furthermore, we performed the globularity prediction to cross check the disorderedness findings, and binding regions of the $N K X 2.5$ (Figure 1E). DISOPRED data shows that residues 10 to 140 in $N K X 2.5$ are highly disorder (above the cutoff value 0.5 ), but the globularity prediction showed that residues 40-140 are globular and may have some linear motif. This result matched well with secondary structure prediction (Supplementary Figure 6) and protein binding motif outcomes. Similarly, in GATA4 the region beyond the core domain residue 210-320 has no globular region and is completely disordered.

From the result of protein binding affinity, disorder tendency analysis and globularity prediction it is clear that although the region TN-domain in $\mathrm{N}$-term and C-term of $N K X 2.5$ are disordered and missing in the reported crystal structures, but may gain secondary structure during the interaction. In $N K X 2.5$, protein binding regions are presents at both $\mathrm{N}$-term and $\mathrm{C}$-term region. While $\mathrm{C}$-term, is reported as an autorepressive domain and its interaction with C-term of GATA4 might induce a conformational change that leads to the unmasking of $N K X 2.5$ activation domain [5]. Since the mutant D16N localizes at N-term and no interactions are yet described for the N-term region. Therefore, to explore the effect of D16N mutant in disease progression, only the $\mathrm{N}$-term and HD regions were modeled for $N K X 2.5$ (region highlighted with black line in Figure 1C). Similarly, in the case of GATA4, protein binding region ware found between $\mathrm{ZnF}$ motif and extended C-term, and this also matched with globularity prediction and consensus secondary structure prediction data (Figure 1F). So, for the present study, only $\mathrm{ZnF}$ domain and extended $\mathrm{C}$-term was modelled and shown for the GATA4 (region highlighted with black line in Figure $1 \mathrm{C})$.

\section{Protein-DNA Docking: DNA binds to HD domain}

The interactions were achieved by protein-DNA docking via NP-dock (http://genesilico.pl/NPDock) and HADDOCK (https://haddock.science.uu.nl) tools (Figure 5 and S2A). From docking results, we found that the residues 141-197 of HD region are mainly participating in interaction with the DNA. The HD residues established the stable hydrogen bonds interacting with DNA are shown in Figure 5 and Supplementary Table 2. The protein-DNA complex was gained additional binding affinity through hydrophobic contacts with residues L144, F145, and I184 and the flexible polar contacts are found with residues R139, P145, R161, R168, I184, W185, and K192. The structural stability of the models was assessed by calculating the evolution time of potential energy and, the root-mean square deviation (RMSD) between $N K X 2.5$ (apo) and NKX2.5-DNA. From the MD simulation we observed that in terms of RMSD values, the NKX2.5-DNA complex is more stable, in which the mean RMSD value (11 $\AA$ ) is lower than $N K X 2.5$ apo-form (14.5 A) (Figure 4C), NKX2.5 moves into more stable
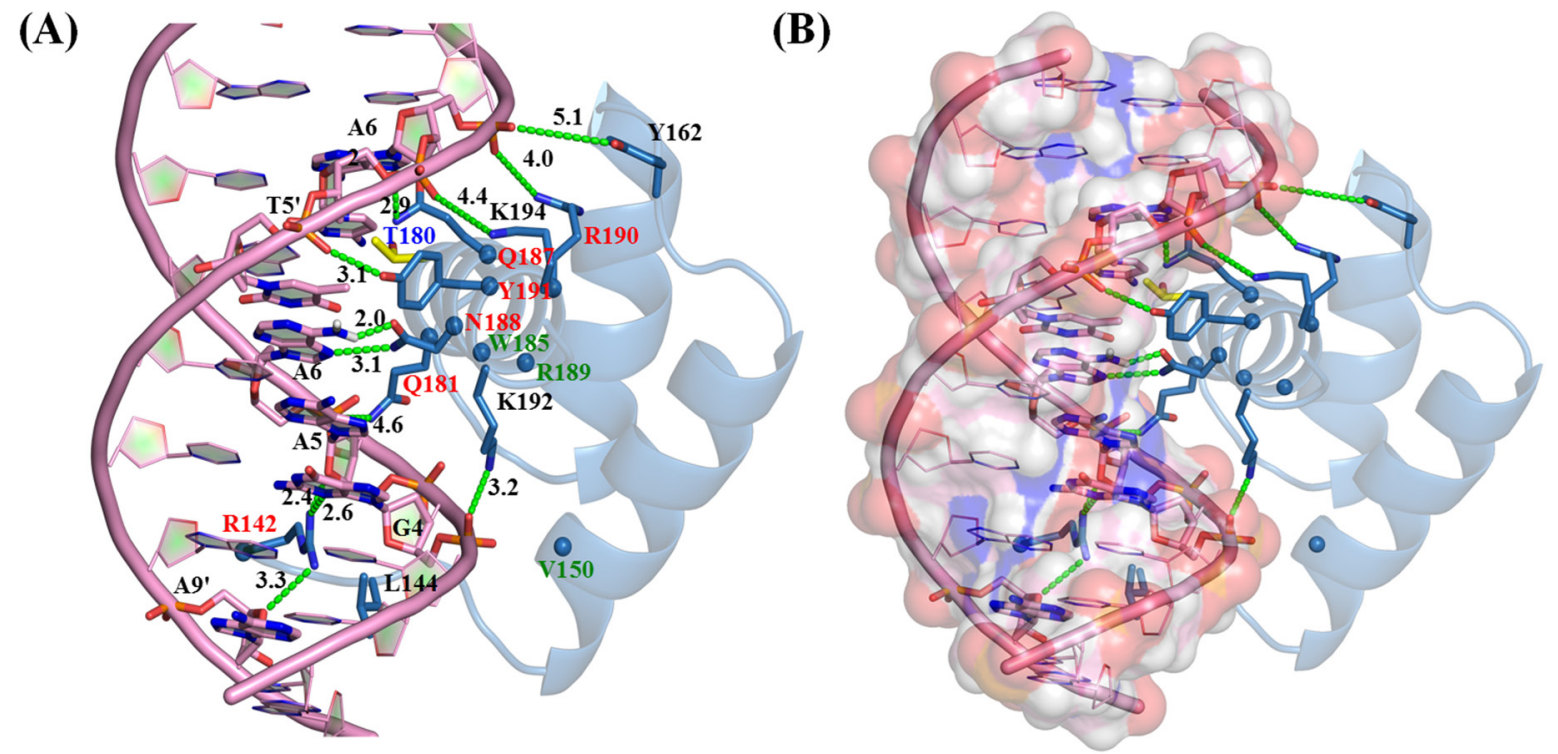

Figure 5: NKX2.5-DNA Interaction map: Residue level interaction between NKX2.5 and DNA (ANF-242). (A) All the residues having interactions with DNA (around $5.0 \AA$ ) are written in four categories: 1) only interacting residues are in black, 2) only mutations are in green color, 3 ) interacting and mutation both residues are red color and, 4) interacting and PTM residue in blue color. (B) DNA and amino acids are rendered as electrostatic surface view and licorice, respectively. 
conformation upon binding of the DNA. Furthermore, we measure the time evolution of distance between $\mathrm{C}$-alpha atom of D16@NKX2.5 and center of mass of nucleotide residue 3 to 7 of chain $C$ and 16 to 19 of chain D of DNA. The mean average distance is $\sim 27 \AA$ and the minimum distance throughout trajectory is $23 \AA$ which is quite high to impact any significant effect of D16N on positioning of DNA (Supplementary Figure 2A, and Supplementary Table 2).

\section{Protein-protein docking: NKX2.5 and GATA4 binds at two binding regions (BR1 and BR2)}

The average structures of $N K X 2.5$ and GATA4 extracted from last $80 \mathrm{~ns}$ of MD simulation were used for protein-protein (PP) docking, respectively. The docked-poses having docking energy above $-30 \mathrm{kcal} /$ mol were selected for best-pose analysis. The top 20 complex poses of NKX2.5 and GATA4, the lowest energy poses (lowest docking energy and maximum number of conformers) was selected for molecular dynamics simulation of complex (Figure 4A and 4B). The interactions achieved after performing the rigid and flexible PP docking shows that amino acid residue 13-38 of $\mathrm{N}$-term of $N K X 2.5$ are interacting with residue 238263 of GATA4 (Figure 6A and 6B and Supplementary Table 3). Prior to MD, the identified interface sites of the complexes were cross-checked by performing the binding site detection method through Schrodinger [36] (Supplementary Figure 7, and Supplementary Table 4). The site detection method identified the BR1 and BR2 as the most prominent interface site, which was corroborated well with the docking outcomes (Supplementary Figure 7). The site score and the volume of the cavity criteria were counted for the selection of the top sites [37].

\section{Molecular dynamics simulation study RMSD}

We build the models of NKX2.5+DNA with GATA4 and $T B X 5$. We find that in partner proteins GATA4 is in the vicinity of D16N, however, TBX5 is far away (Supplementary Figure 8). Therefore, the complex of NKX2.5 and GATA4 was selected for further analysis. The MD simulation was performed for each system: NKX2.5 (apo), NKX2.5-DNA, NKX2.5-GATA4, NKX2.5-DNA-GATA4 (WT) and NKX2.5-DNA-GATA4

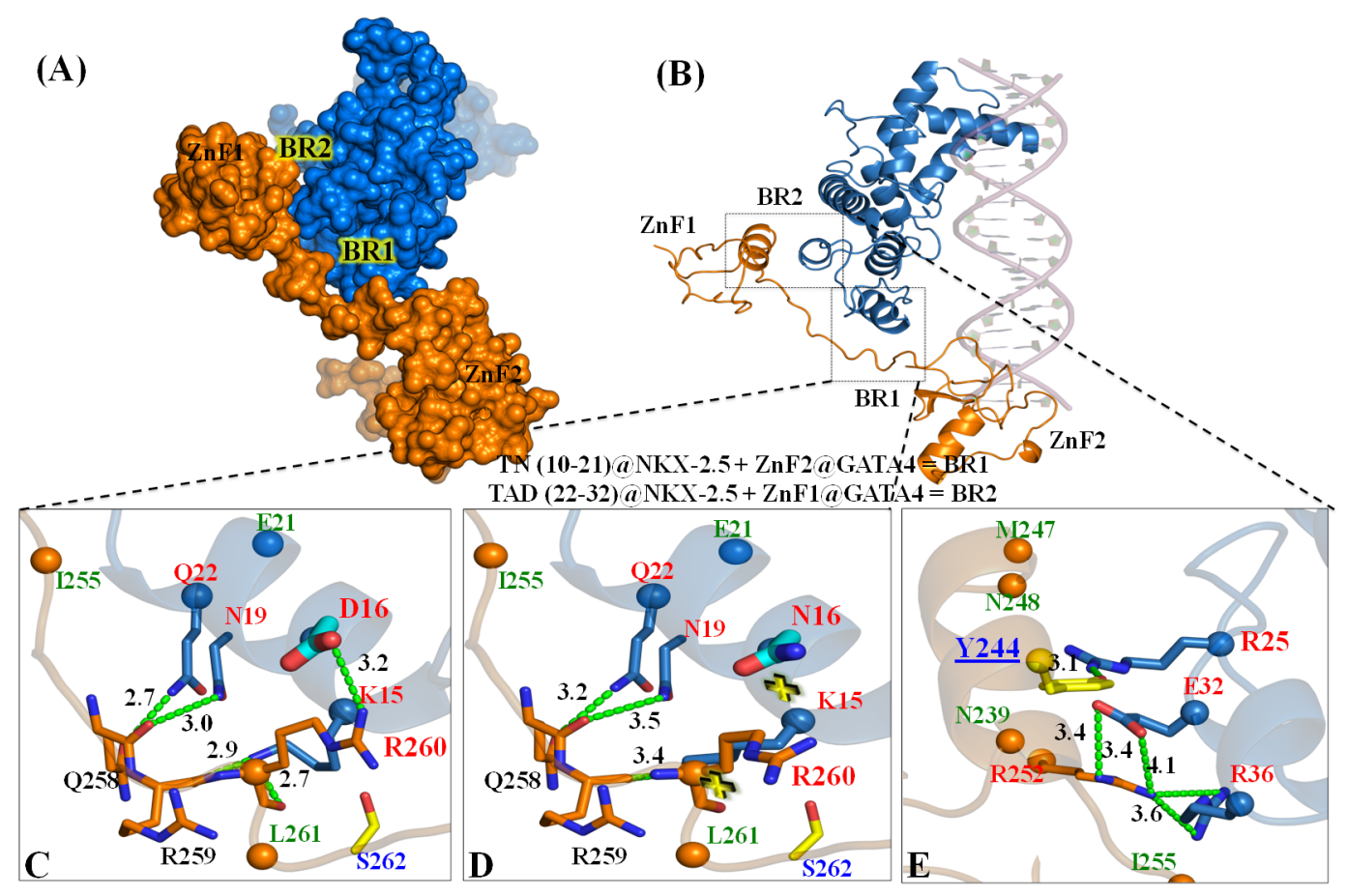

Figure 6: NKX2.5 -GATA4 interaction map: (A) In the interactions zones (BR1 and BR2) of NKX2.5 (in blue surface) and GATA4 (in orange surface) are shown. The binding region constituted by TN (10-21)@NKX2.5+ZnF2@GATA4=BR1, TAD (22-32)@NKX2.5 +ZnF1@GATA4=BR2. (B) The NKX2.5 and GATA4 interaction shown in carton and rendered with same color. The DNA bound with NKX2.5 is shown in dull pink color. The two binding regions (BRs), BR1 and BR2 are highlighted by dotted squares. The zoom-in view, of BR1 is shown in panel (C) and (D), and BR2 is shown in panel (E). In panels (C), (D), and (E), all the key residues are shown as stick and rendered as atom-wise (C: cyan, O: red, N: blue, S: yellow). The residues are categorized in four colors. Red: interacting residues with mutations, Green: only mutation, Black: only interacting and Blue: interacting and PTM. The novel mutation residue D16 (wild-type: in panel C) and N16 (mutant: in panel D) are shown in thick cyan color. (E) The BR2 interaction-map. Zn atom has been omitted from the Figure for the clarity (for Zn atoms see supplementary image S13). 
(MT) (Figure 4C). The system like NKX2.5-DNA (mean RMSD: $11 \AA$ ) and NKX2.5-GATA4 (mean RMSD: 10 $\AA$ ) alone is not dynamically stable, since both have high RMSD $(\geq 10 \AA)$, as in case of NKX2.5-DNA the TN domain is free to move, however, in case of NKX2.5GATA4 the HD domain reflects high flexibility (Figure 4C and 4D). From the RMSD analysis, we find the NKX2.5DNA-GATA4 (WT) is the most stable, in which mean RMSD (2.5 $\AA$ ) is far below than other sets of complexes (Figure 4C). This behavior is clearly reflected in the probability distribution of RMSD of MD systems (Figure 4C) and indicates system NKX2.5 (D16)-DNA-GATA4 (WT) as a true and complete system for the dynamic study of mutation (Figure 4B). The stable WT system is used to check the mutational effect. In WT system the residue D16@NKX2.5 is changed into N16@NKX2.5 to make mutated system, NKX2.5 (N16)-DNA-GATA4 (MT). From the RMSD analysis between system WT and MT, we find a significant $\sim 4.0 \AA$ difference, indicating that the MT system (mean RMSD: $6.5 \AA$ ) dynamics is different than the WT system and a huge decrease in stability occur after D16N mutation.

\section{Domain movement (distance HD-TN)}

Since the $N K X 2.5$ domains (TN+TAD+HD) are essential to hold the DNA (from HD side) and to have interaction with GATA4 (from TN side), as reflected from the dynamics of system NKX2.5-DNA and NKX2.5GATA4, domain movement graph Figure 4D. Therefore, the movement between $\mathrm{HD}$ and $\mathrm{TN}$ is essential to understand the dynamic pattern of the WT and MT systems. From the complex assembly obtained from MD simulation (average structures), the TN domain of $N K X 2.5$ is stably associated with GATA4. Since the mutation D16N, localized in the same $\mathrm{TN}$ domain, therefore, to observe the changes in the dynamics between WT and MT, the center of moss of HD and TN domain was picked for domain movement analysis along the whole trajectories. There were no considerable differences were observed in domain movement of WT and MT complexes (Figure 4D).

\section{Distance analysis at BR1 and BR2 in WT and MT}

Furthermore, to see the effect of D16N mutation, we performed the distance analysis of BR1 and BR2 sites by taking the center of mass of interaction area (Figure 4B\&4F). We observed a significant variation in site BR1 in MT system than the WT system, which pronounced very clearly in the population distribution. In WT the trajectory is distributed with mean value of $5.3 \AA$, while in mutant it is bimodal and also distributed with mean value of $9 \AA$, indicating a remarkable difference at BR1 site (Figure 4F). However, similar to domain movement of HD-TN, the distance at BR2 site is also stable. It indicates that BR1 site severely affected by D16N. The Root mean square fluctuation (RMSF), which measures of the average fluctuation of the residue over time, was also performed to have a clear picture of dynamic changes at BR sites. The RMSF of the $\mathrm{C}_{\mathrm{a}}$ atom of the entire residue over different time frame was plotted to measure the dynamic changes that occur at the level of single residues and analyses the fluctuation of the interface residue of $N K X 2.5$ over the simulation time. The RMSF difference plot was also generated by plotting the difference in RMSF of WT and MT; this was done to determine the regions that have shown the significant structural changes during the MD simulation. The RMSF values clearly indicate that MT has shown significant changes at BR1 compared to the BR2 sites (Figure 4E). The residues that have large fluctuation in MT are 1-25 (BR1), 35-45 (BR2), 79-81, 85-87, 102108 and 134-144. As compared to the BR2 site, the fluctuation at BR1 is very high and this is well represented by the distance time evolution graph also (Figure 4F). The distance time evolution between $\mathrm{Ca}$ atom of residue 16 of NKX2.5 and R260@GATA4 was plotted for system WT and MT (Figure 4G). In case of system WT, the distance is consistent throughout the trajectory with unimodal distribution in probability plot and mean average distance $5.3 \AA$. While, in case of the MT system, distance after 40ns jump to $14 \AA$ and distribution plot is trimodal.

\section{Interaction analysis}

The MD trajectory shows that the complex is stable, and the averaged structure was extracted from the last 80 ns simulation to generate the stable interaction map between NKX2.5 and GATA4. The interface site was quantified in terms of residue-wise interaction energy analysis and, the life-time occupancy of HBs (Figure $4 \mathrm{H}$ and $4 \mathrm{I}$ ). The interaction pattern at the interface site of the complex was quantified in terms of $\mathrm{HBs}$ and $\mathrm{HpH}$ contacts. The HBs formed at BR1 (ZnF2) are NKX2.5@ K15:GATA4@R260,_NKX2.5@K15:GATA4@R259, NKX2.5@D16:GATA4@R260,NKX2.5@N19:GATA4@ Q258 and NKX2.5@Q22:GATA4@Q258 (Figure 6C and 6D). At BR2 ( $\mathrm{ZnF} 1)$ region, the HBs formed are NKX2.5@ R25:GATA4@Y244, and NKX2.5@Q32:GATA4@R252 (Figure 6E). Additionally, the residues strengthen the binding affinity between $N K X 2.5$ (residues V14, I17, L18, L20, L27, A28, A29, A30, L33, A35 and L37) and GATA4 (residues A263, L261, P257, I255, L254, P253, I250, M247, L243, C241 and C238) by establishing the hydrophobic contacts. The HBs that persist longer time are also showing the higher interaction energy (Figure $4 \mathrm{H}$ and 4I), however, the common interaction-life and interaction-energy was found in residues $\mathrm{K} 15$, D16, and R25 of $N K X 2.5$ (Figure 4H and 4I).

\section{Reported pathogenic mutations}

Since we identified a novel pathogenic missense mutation and, from the residue-wise analysis we confirmed that D16 contribute significantly and it involved in the active association of NKX2.5 and GATA4 which 
is essential for their biological function. This provides a clue that those residues that are actively involved in the association of $N K X 2.5$ and GATA4, possibly are the pathogenic mutants. Therefore, the literature was explored with the aim to identify the other pathogenic mutations in NKX2.5 and GATA4.

\section{NKX2.5 HD regions}

We found 18 point-mutations from 15 residues of HD. [10, 11, 38] These mutations are R142, Q149, V150, Q160, R161, Q170, L171, T178, S179, Q181, W185, Q187, N188, R190 and Y191 (Figure 5 and Supplementary Table 5).From the residue wise analysis, we noticed that among these reported pathogenic mutations, most of them are those which are involved in making hydrogen bonds (HBs) with DNA like R142, R161, Q181, W185, N188, R190 and Y191, which underscores the functional importance of this region. Also, we observed that the mutations in amino acids participating in key polar interaction (PI) are more prone to develop into disease state. All the residues having interactions with DNA are analyzed in four categories: (a) only interacting residues either $\mathrm{HBs}$ or hydrophobic $(\mathrm{HpH})(4 / 14)$, (b) only reported pathogenic mutations $(3 / 14)$, (c) interacting and pathogenic both (6/14), and (d) interacting and PTM (1/14). Our docking outcomes corroborate nicely with the previously published crystal data [6]. From the mutation list of HD region and the interaction pattern between DNA and HD, we found that most of the residues are common, indicating that it might be possible that the loss of polar contacts would contribute significantly in the diseased causing state.

\section{Pathogenic mutations in $N K X 2.5$ and $\mathrm{ZnF}$ domain of GATA4}

Similar to HD region, the second hub of the mutations is localized around BR1 and BR2 interface site of $N K X 2.5$ and GATA4. The BR1 site constitutes 3 mutations having interaction with GATA4 residues; while at BR2 site, the total number of 6 mutations is reported. Interestingly, out of 6 mutant residues, 4 same residues are involved either in terms of $\mathrm{HBs}$ or $\mathrm{HpH}$ contacts. (Table 4) Most of the interactions localized at BR sites are transient, indicating that these two proteins come together for a short time as both of them constitute of disordered zone which transformed into ordered form during interactions. Since occurrence of mutations is the well-known cause of disease, therefore, we tried to map all the possible reported mutations in NKX2.5-GATA4 interface (Table 4). Interestingly, most of the known diseases causing mutations are those residues, which we found to contribute significantly in the stability of NKX2.5-GATA4 complex through residue wise analysis. Residue D16@NKX2.5 and R25@NKX2.5 contribute most significantly in stability of NKX2.5-GATA4 complex (Figure 4H and 4I).

\section{Computational mutagenesis and alanine scanning}

The affinity and specificity in protein interface act as a key to regulate/modulate protein-protein interaction. It is a well-known fact that formation of protein-protein complex/s depends on few interface residues contributing most in the binding free energy, called as 'hotspot' [39]. Alanine scanning is the powerful tool to find out binding hotspot at the interface of protein-protein complex. It measures the net change in the binding free energy $\left(\Delta \Delta \mathrm{G}_{\mathrm{b}}\right)$ of a protein-protein complex upon mutation of amino acid residue to alanine. We have scanned all interface residue with Schrodinger module BioLuminate [40], and two other web servers DrugScorePPI [39] and BeatMusic [41]. We performed alanine scanning of all interface residues in both GATA4 and NKX2.5 to find hotspot residues. We found that in all the cases Bioluminate, DrugScorePPI and BeatMusic, the residues, which are forming direct polar interactions, after mutation in alanine, lead to significant decrease in the binding affinity compared to other residues, which are not participating as a polar interaction. Bioluminate identified D16@NKX2.5 $\left(\Delta \Delta \mathrm{G}_{\mathrm{b}}\right.$, $8.65 \mathrm{kcal} / \mathrm{mol})$ as a key residue and have second most decreased binding affinity after R260@GATA4 $\left(\Delta \Delta \mathrm{G}_{\mathrm{b}}\right.$, $16.32 \mathrm{kcal} / \mathrm{mol})$ in alanine scanning of interface residues of both NKX2.5 and GATA4 (Figure 7A, and Table 5 and $6)$. This outcome is well supported by DrugScorePPI $(0.8 \mathrm{kcal} / \mathrm{mol}$, top_score $1.37 \mathrm{kcal} / \mathrm{mol})$ and BeatMusic (0.9 kcal $/ \mathrm{mol}$, top_score $1.29 \mathrm{kcal} / \mathrm{mol}$ ) (Table 5 and 6). The salt-bridges were identified by DrugScorePPI between D16@NKX2.5 and R260@GATA4 at BR1 and, E32@NKX2.5 and R252@GATA4 at BR2. It was reported that the residues involved in salt-bridge formation are considered as key hotspot residue. Furthermore, we also performed the mutagenic analysis for reported mutants at interface through BioLuminate and BeatMusic. The mutagenic analysis corroborates well as it follows the same trend of change of binding free energy as we found in the case of alanine scanning. This analysis confirmed a significant drop of decrease in binding energy especially in residues D16, E32 of NKX2.5 and Y244 and R260 of GATA4 (Supplementary Table 6).

Furthermore, we also performed the Post Translational Modifications (PTMs) identification, as it is known that PTM can induce conformational changes [24, 42], and we find three phosphorylation sites at $\mathrm{ZnF}$ region of GATA4: S262, and S264 at BR2 and Y244 at BR1 sites (Figure 6) [43, 44].

\section{DISCUSSION}

In the present study, we have looked $N K X 2.5$ mutations among 100 CHD patients having ASD, VSD, TOF and SV. Among several reported mutations, we found 
Table 4: Amino acid residues of $N K X 2.5$ and GATA4 present in cutoff value $8.0 \AA$ (cut off taken through pymol)

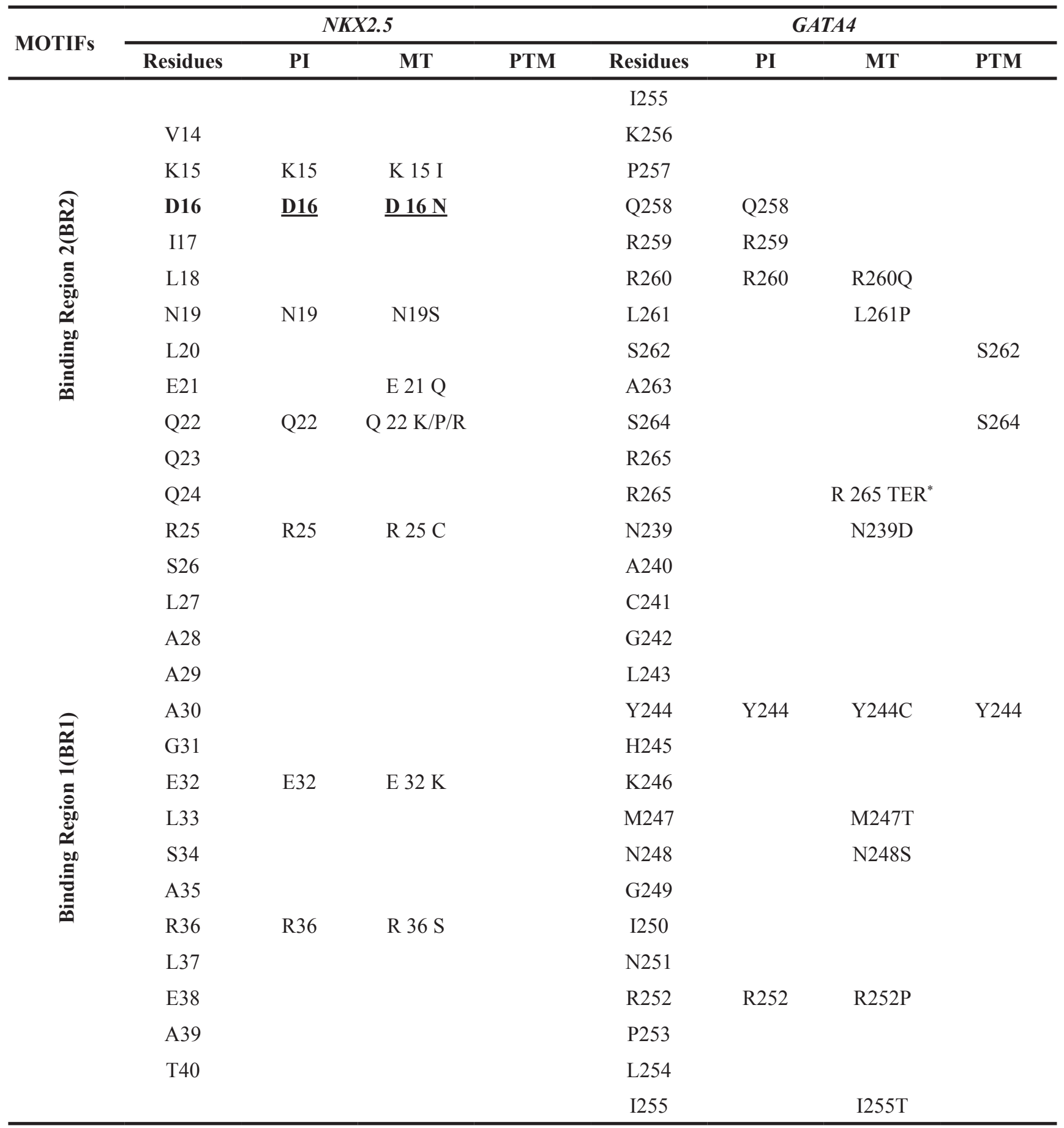

Residues participated in direct polar interaction, mutations, and PTM localize in the interaction zone are listed. PI $=$ Polar Interactions, $\mathrm{MT}=$ Mutations. Bold amino acids are those, which are identified by this work.

one novel missense mutation D16N in CHD patients and associated with a VSD phenotype. This D16N mutation is present in the TN domain of $N K X 2.5$ and conserved in human, cattle, monkey, pig, dog, rat and mouse. (Figure 1B) To know the functional significance of this missense mutation, we performed bioinformatics analysis using PMut, PROVEAN, SIFT, PANTHER, PHD-SNP,
SANP, Polyphen-2 and PredictSNP webserver. PMut, SIFT, PROVEAN, PANTHER, PHD-SNP, SANP, and PredictSNP strongly indicated this mutation is pathogenic. Indeed, PolyPhen-2 act as outlier as it recognizes this D16N mutation as not pathogenic, however, PolyPhen-2 which included in PredictSNP pipeline shows this mutation as pathogenic. The mutation prediction tools 
corroborate nicely with our clinical outcome, motivate us to do further in-depth structural analysis to find localization, molecular mechanism, and its association in perturbed biological function.

The structural and biochemical properties of the disordered regions are ideal for TFs as they can mediate specific recognition of interaction partners or co-ordinate regulatory events in space and time. [45-47] The function of intrinsically disordered proteins may be controlled by some factors like mutations, endo/exogenous ligands and/ or post-translational modifications that lead to structural changes. Previously, no functional role was defined for TN domain in $N K X 2.5$. In this study, we found that amino acid residue D16 present in the $\mathrm{TN}$ domain and playing a crucial role to interact with GATA4. From the protein-protein interaction studies, we identified two hotspots i.e BR1 and BR2. The identification of hot-spots depends on three criteria: a. key interactions, b. reported mutations and, c. localization of PTM sites. Interestingly, we noticed that most of the polar interactions are also reported mutations (Table 4 and S5). It is observed that the localization of D16, and its nearby regions are the hub of CHD mutations in GATA4 and NKX2.5. Additionally, we also identified the phosphorylation site from the database K52@NKX2.5, S262@GATA4, S264@GATA4, and Y244@GATA4 in the vicinity of the interaction sites. Therefore, the key interaction pattern, localization of pathogenic mutations and presence of PTMs at interface site in both proteins, all together justified the identification of two hot-spot BR1 and BR2. The most stable interactions are NKX2.5@D16:GATA4@R260 at BR1 site and NKX2.5@R25:GATA4@Y244 at BR2 site. The residue R260Q@GATA4 is reported as a pathogenic mutation, causing ASD and VSD. [10] Interestingly, NKX2.5@K15 and our identified mutation NKX2.5@ D16 form a stable hydrogen bond with GATA4@R260. The loss of polar contacts with variation at residue R260Q might induce the conformational change and destroy the structural-zip between $N K X 2.5$ and GATA4 at BR1 site and, could be a reason of pathogenicity (Figure $7 \mathrm{~B}$ and 7C). The similar affect possibly reflects if genetic variation occurs at residue D16 (Figure 7B and 7C), indicating the importance of this residue towards pathogenicity. The D16 mutation might be responsible for pathogenicity can be further claimed, by the interaction pattern and biological significance of the residues participating in interaction at BR1 and BR2 site. We found, at the BR2 site, a set of three residues (one acidic and two basic) with one saltbridge (E32@NKX2.5:R252@GATA4) and one hydrogen bond(R36@NKX2.5:R252@GATA4)make an interaction triad. The similar interaction triad pattern, with one saltbridge (D16@NKX2.5:R260@GATA4) and one hydrogen bond (K15@NKX2.5:R260@GATA4), among a set of similar type of three residues (one acidic and two basic) is also formed at the BR1 site. The amino acid variations in the residues of interaction triads at both sites are
(A)

$$
\text { (A) }
$$
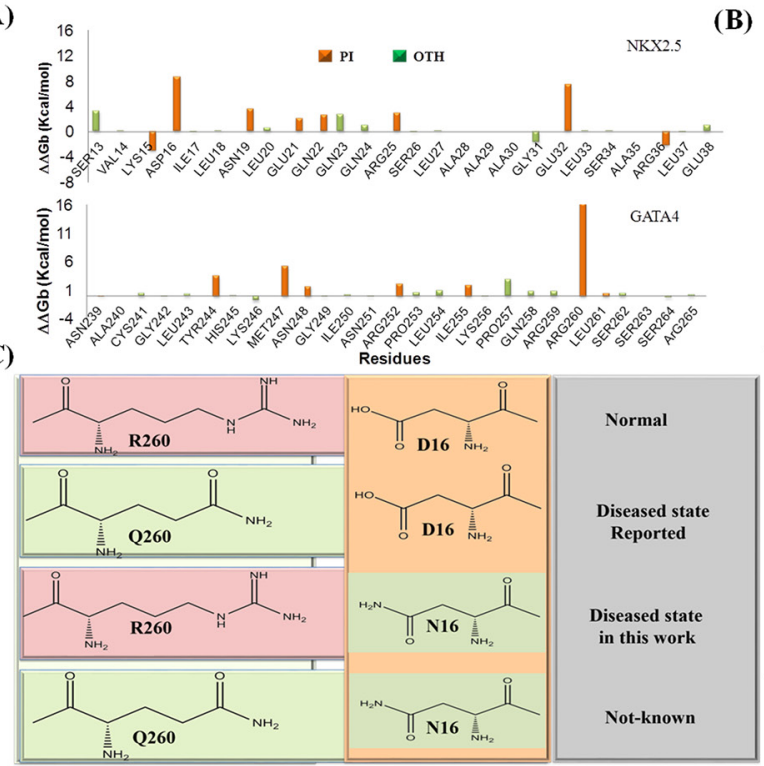

(B)
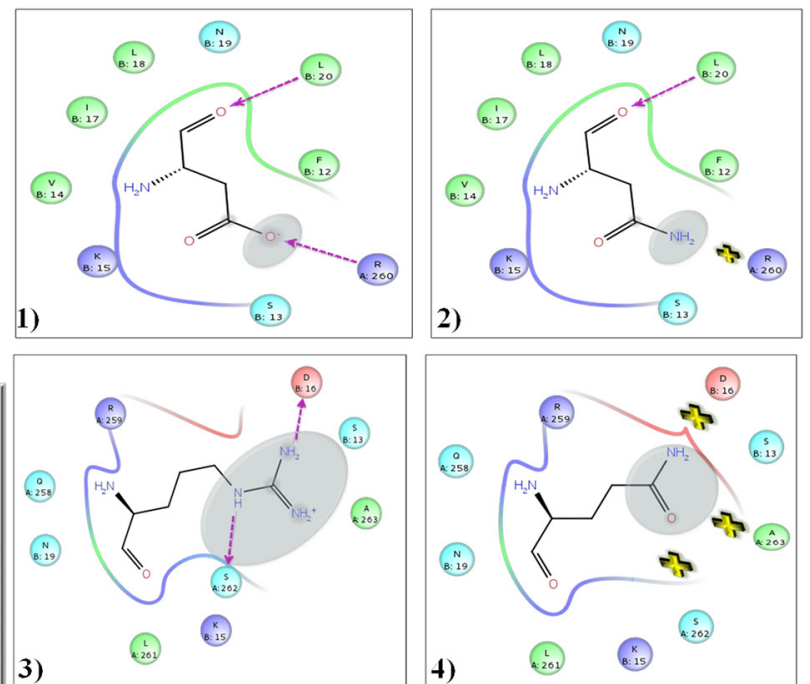

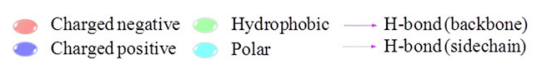

Figure 7: (A) Alanine Scanning: Results summary for BioLuminate alanine scanning of interface residues of NKX2.5-GATA4 protein complex and change in binding free energy/affinity $\left(\Delta \Delta \mathrm{G}_{\mathrm{b}}\right)(\mathrm{kcal} / \mathrm{mol})$. Residue participated in the polar interaction (PI), shown as red bar and rest all other interface residue (OTH), shown as green bar. $\Delta \Delta \mathrm{G}_{\mathrm{b}}$ value higher than zero means decrease in binding affinity and value less than zero means increase in binding affinity. Upper panel belongs to $N K X 2.5$, and lower panel belongs to GATA4. (B) 2D interaction map of D16: 2D map represent the changes in interaction pattern of D16/N16 and R260/Q260 in wild type and Mutant form at BR1 site. (1 and 2) Interaction-map, within $5 \AA$ from $N K X 2.5$ residue: 1) D16; 2) N16. (3 and 4) Interaction-map, within $5 \AA$ from GATA4 residue: 3 ) R260; 4) Q260. (C) Schematic representation: Key residue interactions of D16N@NKX2.5:R260Q@GATA4 and their physiological outcomes. 
Table 5: Results summary for alanine scanning of interface residues of $N K X 2.5$ of NKX2.5-GATA4 protein complex

\begin{tabular}{|c|c|c|c|c|c|}
\hline \multirow{2}{*}{ Residues } & \multirow{2}{*}{$\begin{array}{l}\text { Mutation to } \\
\text { Alanine }\end{array}$} & \multicolumn{3}{|c|}{ Change in binding affinity $\left(\Delta \Delta \mathbf{G}_{\mathrm{b}} \mathrm{kcal} / \mathrm{mol}\right)$} & \multirow{2}{*}{$\begin{array}{l}\text { Possible Salt-bridges } \\
\text { (DrugScorePPI) }\end{array}$} \\
\hline & & BioLuminate & BeatMusic & DrugScorePPI & \\
\hline Ser13 & $\mathrm{K} \rightarrow \mathrm{A}$ & 3.31 & 0.92 & 0.46 & - \\
\hline Val14 & $\mathrm{V} \rightarrow \mathrm{A}$ & 0.08 & 0.03 & 0.06 & - \\
\hline Lys 15 & $\mathrm{~K} \rightarrow \mathrm{A}$ & -3.07 & 0.92 & 1.13 & - \\
\hline Asp16 & $\mathbf{D} \rightarrow \mathbf{A}$ & 8.65 & 0.68 & 0.8 & $\mathbf{X}$ \\
\hline Ile17 & $\mathrm{I} \rightarrow \mathrm{A}$ & -0.03 & 0.08 & - & - \\
\hline Leu18 & $\mathrm{L} \rightarrow \mathrm{A}$ & 0.12 & 0.16 & 0.16 & - \\
\hline Asn19 & $\mathrm{N} \rightarrow \mathrm{A}$ & 3.59 & 1.26 & 1.37 & - \\
\hline Leu20 & $\mathrm{L} \rightarrow \mathrm{A}$ & 0.63 & 0.08 & 0.08 & - \\
\hline Glu21 & $\mathrm{E} \rightarrow \mathrm{A}$ & 2.09 & 0.04 & - & - \\
\hline Gln22 & $\mathrm{Q} \rightarrow \mathrm{A}$ & 2.58 & 0.43 & 0.04 & - \\
\hline Gln23 & $\mathrm{Q} \rightarrow \mathrm{A}$ & 2.78 & 0.28 & 0.54 & - \\
\hline Gln24 & $\mathrm{Q} \rightarrow \mathrm{A}$ & 1.04 & 0.3 & - & - \\
\hline $\operatorname{Arg} 25$ & $\mathrm{R} \rightarrow \mathrm{A}$ & 2.95 & 0.44 & 0.34 & - \\
\hline Ser26 & $\mathrm{S} \rightarrow \mathrm{A}$ & -0.01 & -0.24 & - & - \\
\hline Leu27 & $\mathrm{L} \rightarrow \mathrm{A}$ & 0.02 & 0.3 & - & - \\
\hline Ala28 & - & - & - & - & - \\
\hline Ala29 & - & - & - & - & - \\
\hline Ala30 & - & - & - & - & - \\
\hline Gly31 & $\mathrm{G} \rightarrow \mathrm{A}$ & -1.74 & 0.45 & - & - \\
\hline Glu32 & $\mathrm{E} \rightarrow \mathrm{A}$ & 7.44 & 0.99 & 1.2 & X \\
\hline Leu33 & $\mathrm{L} \rightarrow \mathrm{A}$ & 0.02 & 0.6 & 0.06 & - \\
\hline Ser34 & $\mathrm{S} \rightarrow \mathrm{A}$ & 0.06 & 0.05 & - & - \\
\hline Ala35 & - & - & - & - & - \\
\hline $\operatorname{Arg} 36$ & $\mathrm{R} \rightarrow \mathrm{A}$ & -2.26 & 0.57 & 0.35 & - \\
\hline Leu37 & $\mathrm{L} \rightarrow \mathrm{A}$ & -0.08 & 0.2 & - & - \\
\hline Glu38 & $\mathrm{E} \rightarrow \mathrm{A}$ & 1.02 & -0.09 & - & - \\
\hline
\end{tabular}

The key residues are highlighted. $\Delta \Delta \mathrm{G}_{\mathrm{b}}$ value higher than zero means decrease in binding affinity and value less than zero means increase in binding affinity.

reported as pathogenic [10] except D16 which is reported as pathogenic in this work (Figure 8). Additionally, one more hydrogen bond R25@NKX2.5:Y244@GATA4 is identified at the BR2 site and, the amino acid mutations R25C@NKX2.5 and Y244C@GATA4 are reported as a pathogenic diseased state [10]. From the structural modeling data, we found that these interaction patterns would be lost due to variation at any of these amino acids. Thus the mutation/s at these amino acids possibly disturbs the interface interaction map which leads to the unstability of the complex.

Other residues of $N K X 2.5$ (N19 and Q22) and GATA4 (Q258 and R259) are also contributing in zipping the NKX2.5-GATA4 interactions at BR1 and BR2 sites by making hydrogen bonds. In general, these two TFs are mostly unstructured disordered form. For regulation of transcription, these two TFs come close together at some binding sites and the binding regions are transformed from unstructured-to-structured form. It seems that the hydrogen bonds between K15, D16, N19, Q21 and R25 of NKX2.5 and Y244, Q258, R259 and R260 of GATA4 at BR1 and BR2 sites are acting like a "zip" to hold the interactions at these two sites. The regions of GATA4 which interact with D16@NKX2.5 are unstructured, flexible and have two reported phosphorylation sites i.e. S262 and S264. The phosphorylation at these 
Table 6: Results summary for alanine scanning of interface residues of GATA4 of NKX2.5-GATA4 protein complex

\begin{tabular}{|c|c|c|c|c|c|}
\hline \multirow{2}{*}{ Residues } & \multirow{2}{*}{ Mutation to Alanine } & \multicolumn{3}{|c|}{ Change in binding affinity $\left(\Delta \Delta G_{b} \mathrm{kcal} / \mathrm{mol}\right)$} & \multirow{2}{*}{$\begin{array}{c}\text { Possible Salt-bridges } \\
\text { (DrugScorePPI) }\end{array}$} \\
\hline & & BioLuminate & BeatMusic & DrugScorePPI & \\
\hline Asn239 & $\mathrm{N} \rightarrow \mathrm{A}$ & 0.1 & -0.03 & -0.06 & - \\
\hline Ala240 & - & - & - & - & - \\
\hline Cys241 & $\mathrm{C} \rightarrow \mathrm{A}$ & 0.52 & 0.34 & 0.28 & - \\
\hline Gly 242 & $\mathrm{G} \rightarrow \mathrm{A}$ & -0.04 & 0.04 & - & - \\
\hline Leu243 & $\mathrm{L} \rightarrow \mathrm{A}$ & 0.29 & 0.18 & 0.27 & - \\
\hline Tyr244 & $\mathbf{Y} \rightarrow \mathbf{A}$ & 3.61 & 1.72 & 0.55 & - \\
\hline His 245 & $\mathrm{H} \rightarrow \mathrm{A}$ & 0.12 & 0.20 & - & - \\
\hline Lys 246 & $\mathrm{~K} \rightarrow \mathrm{A}$ & -0.78 & 0.11 & - & - \\
\hline Met247 & $\mathrm{M} \rightarrow \mathrm{A}$ & 5.28 & 0.51 & 0.19 & - \\
\hline Asn248 & $\mathrm{N} \rightarrow \mathrm{A}$ & 1.79 & 0.43 & 0.32 & - \\
\hline Gly249 & $\mathrm{G} \rightarrow \mathrm{A}$ & -0.02 & -0.13 & - & - \\
\hline Ile250 & $\mathrm{I} \rightarrow \mathrm{A}$ & 0.18 & 0.58 & 0.67 & - \\
\hline Asn251 & $\mathrm{N} \rightarrow \mathrm{A}$ & -0.09 & 0.23 & 0.07 & - \\
\hline Arg252 & $\mathrm{R} \rightarrow \mathrm{A}$ & 2.18 & 1.41 & 0.45 & X \\
\hline Pro 253 & $\mathrm{P} \rightarrow \mathrm{A}$ & 0.62 & 0.32 & - & - \\
\hline Leu254 & $\mathrm{L} \rightarrow \mathrm{A}$ & 0.98 & 0.16 & 0.16 & - \\
\hline Ile255 & $\mathrm{I} \rightarrow \mathrm{A}$ & 1.98 & 0.52 & 0.52 & - \\
\hline Lys 256 & $\mathrm{~K} \rightarrow \mathrm{A}$ & -0.13 & 0.00 & 0.05 & - \\
\hline Pro257 & $\mathrm{P} \rightarrow \mathrm{A}$ & 2.85 & 0.27 & - & - \\
\hline $\mathrm{G} \ln 258$ & $\mathrm{Q} \rightarrow \mathrm{A}$ & 0.82 & 0.63 & 0.37 & - \\
\hline Arg259 & $\mathrm{R} \rightarrow \mathrm{A}$ & 0.86 & 0.15 & 0.01 & - \\
\hline Arg260 & $\mathbf{R} \rightarrow \mathbf{A}$ & 16.32 & 1.77 & 1.70 & $\mathbf{X}$ \\
\hline Leu261 & $\mathrm{L} \rightarrow \mathrm{A}$ & 0.53 & -0.06 & 0.07 & - \\
\hline Ser262 & $\mathrm{S} \rightarrow \mathrm{A}$ & 0.47 & 0.46 & 0.26 & - \\
\hline Ser263 & $\mathrm{S} \rightarrow \mathrm{A}$ & 0.34 & 0.46 & - & - \\
\hline Ser264 & $\mathrm{S} \rightarrow \mathrm{A}$ & -0.24 & 0.25 & - & - \\
\hline Arg265 & $\mathrm{R} \rightarrow \mathrm{A}$ & 0.22 & 0.19 & - & - \\
\hline
\end{tabular}

The key residues are highlighted. $\Delta \Delta \mathrm{G}_{\mathrm{b}}$ value higher than zero means decrease in binding affinity and value less than zero means increase in binding affinity.

sites might induce the conformational change and possibly play a role in on/off switching mechanism pattern during interaction between NKX2.5-GATA4. The phosphorylation sites are known to induce some conformational changes and helping to regulate transient interactions specially at interfaces and binding hot-spot. $[48,49]$ Result of residue wise analysis and in-silico alanine scanning mutagenesis have given clue that the interface site BR1 and BR2 act as binding hot spot, and residue D16@NKX2.5 act as a key hot spot residue. Interestingly, except D16, all other residues are reported as pathogenic mutations indicating the importance of $\mathrm{BR} 1$ and BR2 sites for transcription regulation (Figure 6 and 7). The genetic variations of BR sites residues have already been documented to perturb the transcriptional regulation. We have noticed that D16 residue directly perturb the hydrogen bond with R260 of GATA4 and parallel hindering other key interactions too. These alterations at residue level induce significant structural conformation-orientation changes and loss of secondary structures, by which it unzips the binding of $N K X 2.5$ and GATA4 at BR sites and induces perturbed transcription 
regulation. Therefore, we concluded that D16N mutation of $N K X 2.5$ might be pathogenic as the variation in this residue, not only become a cause of losing the hydrogen bond with R260 of GATA4, also perturbing the interaction pattern at BR1 and BR2 sites significantly. The perturbation of this interaction may impair the downstream pathways crucial for heart development. It is reported that protein $N K X 2.5$, has an autoinhibitory domain and, alone does not have significant effects on the expression of downstream target genes such as A1 adenosine receptor, $\alpha$-cardiac actin, cardiac troponin $\mathrm{T}$, cardiac ankyrin repeat protein, leucine-rich repeat containing 10 and inhibitor of differentiation/DNA binding-2. Direct physical-interaction of $N K X 2.5$ with GATA4 is crucial to regulate the expression of the above essential cardiac genes in a synergistic manner $[5,50$, 51]. It is well established that $N K X 2.5$ cooperatively with GATA4 facilitates its activating and repressing functions. The interaction between NKX2.5 and GATA4 might also be important for the function as a repressor of ion channels and its downstream target genes. Therefore, We hypothesized that the mutation D16N possibly destroy or lose the interaction between $N K X 2.5$ and GATA4 and thus may altered the transcription regulation and eventually leads to loss of several activator and repressor function of this complex.

In summary, we have identified a novel $N K X 2.5$ genetic variation, D16N which is associated with disease VSD in South Indian patients. This work underscores the importance of interaction sites which is the hub of pathogenic mutations. We have identified some key residues from $N K X 2.5$ (Y162, K192, and K194) and GATA4 (Q258 and R259), which contributes substantially in NKX2.5 and GATA4 complex assembly. We have also found two phosphorylation (S262, and S264) sites in the vicinity of the interaction site that might be responsible for conformational changes, as a prerequisite for TFs regulation. The genetic variations at these amino acids possibly alter the function of the proteins. To further confirm, there is a need to make a genetic mouse model with $N K X 2.5$ D16 mutation and development of CHD phenotype. Overall, this work highlights a novel pathogenic mutation D16N in NKX2.5 causing VSD and, underscores the structural mechanistic as how D16N can induce the structural-functional divergence that possibly leads to the disease state.

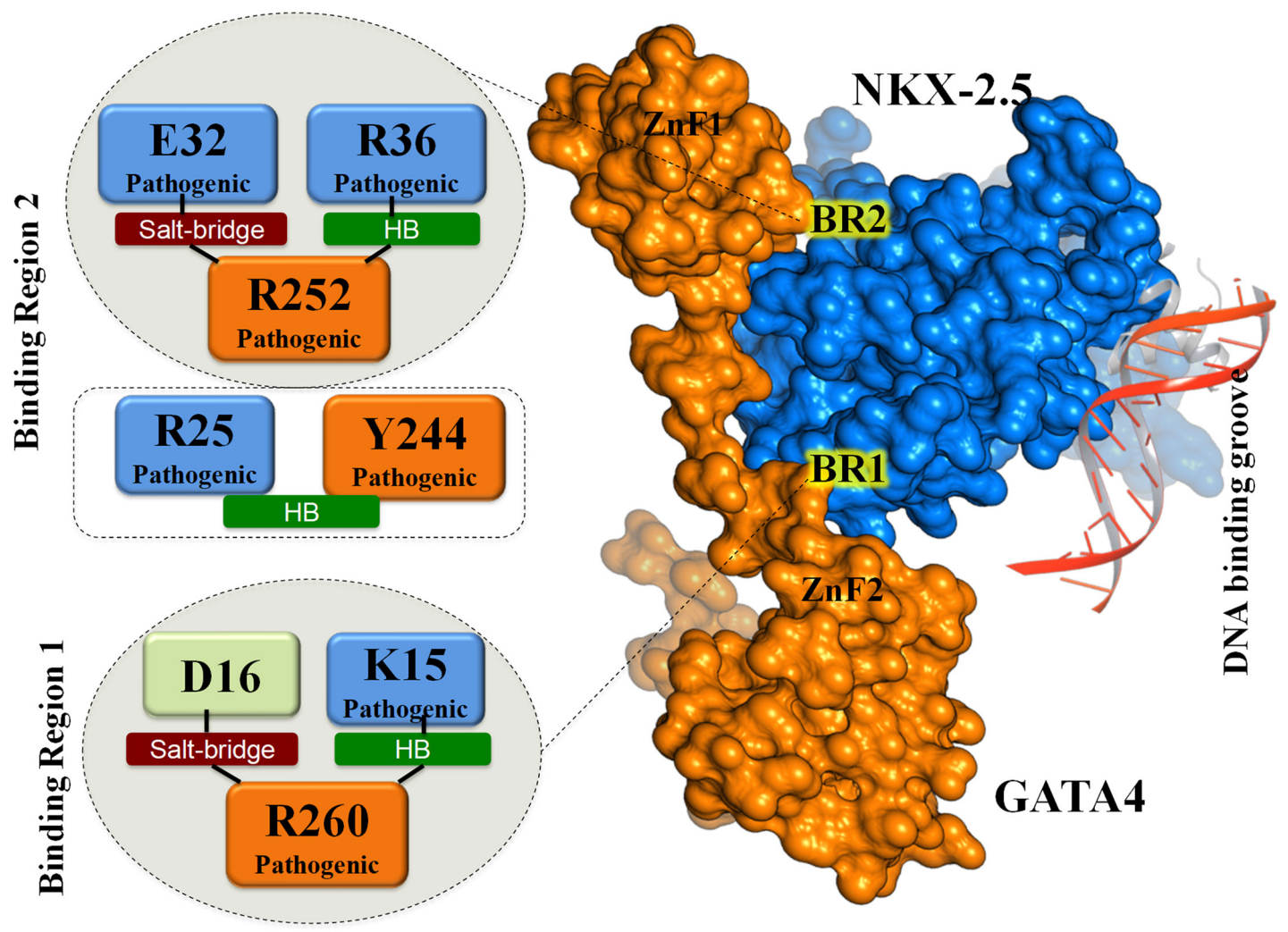

Figure 8: Biological significance of identified key residues: The key interaction triads (in form of salt-bridges and HBs) are highlighted at identified site of BR2 (ZnF1) and BR1 (ZnF2). Except residue D16 (in lime color), all other residues of NKX2.5 (in blue) and GATA4 (in orange) are reported as "pathogenic mutations". 


\section{MATERIALS AND METHODS}

\section{Clinical evaluation and sample collection}

In this study, we used two different selection criteria during collection of sample. The first criteria were CHD types such (ASD, VSD, TOF and SV). The numbers of CHD patient diagnosed in south part of India are much more in these four categories. The second criteria were demographics i.e. selection of the study population made according to geographical location, speaking the Dravidian language (Dravidians) and living in southern India. By considering both selection approaches we collected samples of CHD patients and control group. All the CHD patients studied $(\mathrm{n}=100)$ were outpatients and were recruited at the Innova Children's Heart Hospital in Hyderabad, India. Control samples $(n=200)$, who belonged to the same ethnicity, were collected at Innova Children's Heart Hospital and other hospitals in Hyderabad. Patients were clinically evaluated by predesigned protocol that includes 2D echocardiography, color doppler and ECG. Atrial septal defect (ASD), ventricular septal defect (VSD), tetralogy of fallot (TOF), and single ventricle (SV) were considered for the present investigation. Informed written consent was received from all CHD patients and control samples. The study was approved by the research advisory committee and institutional ethical committee of Innova Children's Heart Hospital (IEC/IRB No. 001/2010), Hyderabad, India.

\section{Genotyping}

DNA was isolated from blood samples according to the protocol of Sambrook et al., 1989. [52] NKX2.5 sequence from the ENSEMBL (ID: ENSG00000183072; www.ensembl.org) was used to design primer employing primer 3 software (http://frodo.wi.mit.edu/) and synthesized commercially (Eurofins, India). We investigated the genomic DNA of CHD patients for variations in the entire coding and untranslated regions ( 3 ' UTR and 5' UTR) of NKX2.5 gene. Detailed sequences of all primers used in this study have been summarized in Supplementary Table 7. We performed PCR, sequencing and details methods followed according to mattapally et al, 2015. [19]

\section{Mutation analysis}

The raw sequence data were analyzed and carefully edited using the sequence analysis software. The edited sequences were assembled with reference sequence using DNA Star and Autoassembler software (Applied Biosystems, USA). All the variant sites, compared to the reference sequence were noted down. Genetic Association, Hardy-Weinberg equilibrium and Chi-square test were computed by using Plink software. Multiple alignments of NKX2.5 amino acid sequences from different species across mammals were done by ClustalX [53]. Pathogenic potential of identified missense mutations from CHD patients was predicted by different webservers such as SIFT, PMut, PROVEAN, PANTHER, PHD-SNP, SNAP, polyphen2 (http://genetics.bwh.harvard.edu/pph2/), and PredictSNP.

\section{Statistical analysis}

Statistical analysis was performed with Plink software and level of significance was set to a p-value of 0.05 . The significance of deviations from Hardy-Weinberg equilibrium was tested using Plink software, we used cut off p-value 0.05; and for association analysis, we used same cut off $p$-value (0.05). We did association analysis and generated pictures with $\mathrm{R}$ basic packages ( $\mathrm{R}$ version 3.0.2, 2013).

\section{Molecular modeling study}

To conduct the 3D model of $N K X 2.5$, the BLAST (from NCBI) was performed with the aim to identify the existing crystal structures with high identity and similarity. Additionally, we performed disorder tendency and protein binding analysis through DISOPRED, which highlight the probability estimation of each residue in the sequence. All details of the molecular modeling of the NKX2.5 model (N-term + HD) and GATA4 model were given in the material and method section in supplementary file. We have also described the method part of, finding of NKE motif in ANF promoter and Preparation of DNA, protein-DNA docking (NKX2.5DNA), protein-protein docking (P-P docking) of NKX2.5-GATA4 complex in details in the material and method section in supplementary file.

\section{Molecular dynamics simulation}

Molecular dynamic (MD) study for all complexes was carried out using Desmond. [54] The molecular systems were first built using System Builder panel. The parameters were assigned from inbuilt OPLS3 force field. "TIP3P" water model [55] was used to solvate the systems using orthorhombic box with distance of $10 \AA$ from all sides of protein complex [56]. System was electrically neutralized by adding appropriate counter $\mathrm{Na}+/ \mathrm{Cl}$ - ions . The prepared systems were further minimized using steepest descent algorithm for 2000 iterations with convergence threshold of $1 \mathrm{kcal} / \mathrm{mol} / \AA$. The minimized systems were then equilibrated using default algorithm which included two stages of minimization (restrained and unrestrained) followed by four stages of MD runs with gradually diminishing restraints using NVT at $300 \mathrm{~K}$, NVT at $700 \mathrm{~K}, \mathrm{NPT}$ at $300 \mathrm{~K}$, and NPT at $300 \mathrm{~K}$ respectively. 
Finally, 50 ns of MD simulation was performed using NPT ensemble wherein Nose-Hoover Chain thermostat [57] was used for temperature coupling at $300 \mathrm{~K}$ and MartynaTobias-Klein barostat [58] for pressure coupling at $1 \mathrm{~atm}$. Long range coulombic interactions were calculated using smooth Particle-Mesh-Ewald method [59] while a cutoff of $9.0 \AA$ for short range electrostatics contributions. Coordinates and energy were recorded every 10 ps to yield 5000 frames.

\section{Computational mutagenesis and alanine scanning}

Alanine scanning mutagenesis was carried out through Schrodinger BioLuminate tool and web servers, DrugScorePPI and BeatMusic. The PDB file of NKX2.5-GATA4 complex was prepared and submitted to DrugScorePPI and BeatMusic webserver and the chain of interest was selected for alanine scanning. The residues scanning in BioLuminate was performed through residue scanning/affininty maturation panel in biologics with refinement option set to side-chain prediction and backbone minimization, the cutoff distance was set to 0.0. BioLuminate, DrugScorePPI and BeatMusic all calculate the net change in binding affinity $(\Delta \Delta \mathrm{Gb})$ produced by single point mutation. Schrodinger module BioLuminate utilizes MM-GBSA approach with force field OPLS2005 and solvent model VSGB to calculate $\Delta \Delta \mathrm{Gb}$. DrugScorePPI, work on knowledge-based scoring function and automatically detects all interface residues within $5 \AA$ cutoff and mutate them one by one to alanine and calculate $\Delta \Delta \mathrm{G}_{\mathrm{b}}$. BeatMusic depends on the residuebased set of statistical potential obtained from known protein structure.

\section{Author contributions}

S.M. and M.S. contributed equally to this work. S.A. and S.K.B. participated in design and coordinated the research. S.M. participated in the sample collection and conducted biological experiments and M.S. done the computational modeling and in-silico parts. K.S.M has participated in the selection of studied patients and carried out diagnosis of patient. S.K.B. and S.A. analyzed the data. S.M., M.S., S.K.B. and S.A. wrote the manuscript. The authors read and approved the manuscript.

\section{ACKNOWLEDGMENTS}

S.K.B is thankful to DBT for providing Ramalingaswamy fellowship. S.M is thankful to Indian Council of Medical Research (ICMR), New Delhi for awarding Junior/Senior Research Fellowship. M.S. is thankful to Council of Scientific \& Industrial Research (CSIR), New Delhi for awarding Senior Research Fellowship. S.K.B. and S.A. are thankful to DDRC (THSTI) for providing necessary support.

\section{Supplementary information}

Supplemental information includes supplemental text, Supplementary Table 1-7, and Supplementary Figures $1-9$.

Supplemental text:

$>$ Method: Modeling of the NKX2.5, modeling of GATA4, Finding of NKE motif in ANF promoter and Preparation of DNA, Protein-DNA docking, Protein-Protein Docking (P-P docking) of NKX2.5-GATA4.

$>$ RESULT: Modeling of NKX2.5, modeling of GATA4, Disorder, globularity and binding region prediction in GATA4 and Explanation of ERRAT.

\section{CONFLICTS OF INTEREST}

The authors declare that they do not have any conflicts of interest.

\section{REFERENCES}

1. Shiojima I, Komuro I, Inazawa J, Nakahori Y, Matsushita I, Abe T, Nagai R, Yazaki Y. Assignment of cardiac homeobox gene CSX to human chromosome 5q34. Genomics. 1995; 27:204-6.

2. Harvey RP. NK-2 homeobox genes and heart development. Dev Biol. 1996; 178:203-16.

3. Apergis GA, Crawford N, Ghosh D, Steppan CM, Vorachek WR, Wen P, Locker J. A novel nk-2-related transcription factor associated with human fetal liver and hepatocellular carcinoma. J Biol Chem. 1998; 273:2917-25.

4. Hiroi Y, Kudoh S, Monzen K, Ikeda Y, Yazaki Y, Nagai R, Komuro I. Tbx5 associates with Nkx2-5 and synergistically promotes cardiomyocyte differentiation. Nat Genet. 2001; 28:276-80.

5. Durocher D, Charron F, Warren R, Schwartz RJ, Nemer M. The cardiac transcription factors Nkx2-5 and GATA-4 are mutual cofactors. EMBO J. 1997; 16:5687-96.

6. Pradhan L, Genis C, Scone P, Weinberg EO, Kasahara H, Nam HJ. Crystal structure of the human NKX2. 5 homeodomain in complex with DNA target. Biochemistry. 2012; 51:6312-9.

7. Tanaka M, Chen Z, Bartunkova S, Yamasaki N, Izumo S. The cardiac homeobox gene Csx/Nkx2.5 lies genetically upstream of multiple genes essential for heart development. Development. 1999; 126:1269-80.

8. Lyons I, Parsons LM, Hartley L, Li R, Andrews JE, Robb L, Harvey RP. Myogenic and morphogenetic defects in the heart tubes of murine embryos lacking the homeo box gene Nkx2-5. Genes Dev. 1995; 9:1654-66.

9. Targoff KL, Colombo S, George V, Schell T, Kim SH, Solnica-Krezel L, Yelon D. Nkx genes are essential for maintenance of ventricular identity. Development. 2013; 140:4203-13. 
10. Stenson PD, Ball EV, Mort M, Phillips AD, Shiel JA, Thomas NS, Abeysinghe S, Krawczak M, Cooper DN. Human Gene Mutation Database (HGMD): 2003 update. Hum Mutat. 2003; 21:577-81.

11. Muntean I, Toganel R, Benedek T. Genetics of Congenital Heart Disease: Past and Present. Biochem Genet. 2017; 55: 105-123.

12. Reamon-Buettner SM, Sattlegger E, Ciribilli Y, Inga A, Wessel A, Borlak J. Transcriptional defect of an inherited NKX2-5 haplotype comprising a SNP, a nonsynonymous and a synonymous mutation, associated with human congenital heart disease. PLoS One. 2013; 8:e83295.

13. Chung IM, Rajakumar G. Genetics of congenital heart defects: the NKX2-5 gene, a key player. Genes. 2016; 7:6.

14. Pierpont ME, Basson CT, Benson DW Jr, Gelb BD, Giglia TM, Goldmuntz E, McGee G, Sable CA, Srivastava D, Webb CL, and American Heart Association Congenital Cardiac Defects Committee, Council on Cardiovascular Disease in the Young. Genetic basis for congenital heart defects: current knowledge: a scientific statement from the American Heart Association Congenital Cardiac Defects Committee, Council on Cardiovascular Disease in the Young: endorsed by the American Academy of Pediatrics. Circulation. 2007; 115:3015-38.

15. Jenkins KJ, Correa A, Feinstein JA, Botto L, Britt AE, Daniels SR, Elixson M, Warnes CA, Webb CL, American Heart Association Council on Cardiovascular Disease in the Young. Noninherited risk factors and congenital cardiovascular defects: current knowledge: a scientific statement from the American Heart Association Council on Cardiovascular Disease in the Young: endorsed by the American Academy of Pediatrics. Circulation. 2007; 115:2995-3014

16. Lloyd-Jones D, Adams R, Carnethon M, De Simone G, Ferguson TB, Flegal K, Ford E, Furie K, Go A, Greenlund K, Haase N, Hailpern S, Ho M, et al, and American Heart Association Statistics Committee and Stroke Statistics Subcommittee. Heart disease and stroke statistics - 2009 update: a report from the American Heart Association Statistics Committee and Stroke Statistics Subcommittee. Circulation. 2009; 119:e21-181.

17. Minette MS, Sahn DJ. Ventricular septal defects. Circulation. 2006; 114:2190-7.

18. Taksande A, Vilhekar K, Chaturvedi P, Jain M. Congenital malformations at birth in Central India: A rural medical college hospital based data. Indian J Hum Genet. 2010; 16:159-63.

19. Mattapally S, Nizamuddin S, Murthy KS, Thangaraj K, Banerjee SK. c. $620 \mathrm{C}>\mathrm{T}$ mutation in GATA4 is associated with congenital heart disease in South India. BMC Med Genet. 2015; 16:7.

20. Pradhan L, Gopal S, Li S, Ashur S, Suryanarayanan $\mathrm{S}$, Kasahara H, Nam HJ. Intermolecular Interactions of Cardiac Transcription Factors NKX2.5 and TBX5. Biochemistry. 2016; 55:1702-10.
21. Mathelier A, Zhao X, Zhang AW, Parcy F, Worsley-Hunt R, Arenillas DJ, Buchman S, Chen CY, Chou A, Ienasescu H, Lim J, Shyr C, Tan G, et al. JASPAR 2014: an extensively expanded and updated open-access database of transcription factor binding profiles. Nucleic Acids Res. 2014; 42:D142-7.

22. Ward JJ, Sodhi JS, McGuffin LJ, Buxton BF, Jones DT. Prediction and functional analysis of native disorder in proteins from the three kingdoms of life. J Mol Biol. 2004; 337:635-45.

23. Costantini S, Facchiano AM, Colonna G. Evaluation of the structural quality of modeled proteins by using globularity criteria. BMC Struct Biol. 2007; 7:9.

24. Bah A, Vernon RM, Siddiqui Z, Krzeminski M, Muhandiram R, Zhao C, Sonenberg N, Kay LE, Forman-Kay JD. Folding of an intrinsically disordered protein by phosphorylation as a regulatory switch. Nature. 2015; 519:106-9.

25. Luna-Zurita L, Stirnimann CU, Glatt S, Kaynak BL, Thomas S, Baudin F, Samee MA, He D, Small EM, Mileikovsky M, Nagy A, Holloway AK, Pollard KS, et al. Complex Interdependence Regulates Heterotypic Transcription Factor Distribution and Coordinates Cardiogenesis. Cell. 2016; 164:999-1014.

26. Wright PE, Dyson HJ. Intrinsically disordered proteins in cellular signalling and regulation. Nat Rev Mol Cell Biol. 2015; 16:18-29.

27. Meszaros B, Simon I, Dosztanyi Z. Prediction of protein binding regions in disordered proteins. PLoS Comput Biol. 2009; 5:e1000376.

28. Laskowski RA, MacArthur MW, Moss DS, Thornton JM. PROCHECK: a program to check the stereochemical quality of protein structures. J Appl Cryst. 1993; 26:283-91.

29. Durham E, Dorr B, Woetzel N, Staritzbichler R, Meiler J. Solvent accessible surface area approximations for rapid and accurate protein structure prediction. J Mol Model. 2009; 15:1093-108.

30. MacArthur MW, Laskowski RA, Thornton JM. Knowledgebased validation of protein structure coordinates derived by X-ray crystallography and NMR spectroscopy. Curr Opin Struct Biol. 1994; 4:731-7.

31. Wiederstein M, Sippl MJ. ProSA-web: interactive web service for the recognition of errors in three-dimensional structures of proteins. Nucleic Acids Res. 2007; 35:W407-W10.

32. Webb B, Sali A. Protein structure modeling with MODELLER. Protein Structure Prediction. 2014:1-15.

33. Ward JJ, McGuffin LJ, Bryson K, Buxton BF, Jones DT. The DISOPRED server for the prediction of protein disorder. Bioinformatics. 2004; 20:2138-9.

34. Dosztanyi Z, Meszaros B, Simon I. ANCHOR: web server for predicting protein binding regions in disordered proteins. Bioinformatics. 2009; 25:2745-6.

35. Puntervoll $\mathrm{P}$, Linding R, Gemund $\mathrm{C}$, Chabanis-Davidson S, Mattingsdal M, Cameron S, Martin DM, Ausiello G, Brannetti B, Costantini A, Ferre F, Maselli V, Via A, et 
al. ELM server: A new resource for investigating short functional sites in modular eukaryotic proteins. Nucleic Acids Res. 2003; 31:3625-30.

36. Discovery Informatics Suite 2016-3. New York (NY): Schrödinger, LLC. 2016.

37. Asthana S, Shukla S, Vargiu AV, Ceccarelli M, Ruggerone P, Paglietti G, Marongiu ME, Blois S, Giliberti G, La Colla P. Different molecular mechanisms of inhibition of bovine viral diarrhea virus and hepatitis C virus RNA-dependent RNA polymerases by a novel benzimidazole. Biochemistry. 2013; 52:3752-64.

38. Consortium U, and UniProt Consortium. The universal protein resource (UniProt). Nucleic Acids Res. 2008; 36:D190-95.

39. Krüger DM, Gohlke H. DrugScorePPI webserver: fast and accurate in silico alanine scanning for scoring proteinprotein interactions. Nucleic Acids Res. 2010; 38:W480-86.

40. Biologics Suite 2017-2: BioLuminate, Schrödinger, LLC, New York, NY. 2017.

41. Dehouck Y, Kwasigroch JM, Rooman M, Gilis D. BeAtMuSiC: prediction of changes in protein-protein binding affinity on mutations. Nucleic Acids Res. 2013; 41:W333-39.

42. van der Lee R, Lang B, Kruse K, Gsponer J, Sanchez de Groot N, Huynen MA, Matouschek A, Fuxreiter M, Babu MM. Intrinsically disordered segments affect protein half-life in the cell and during evolution. Cell Rep. 2014; 8:1832-44.

43. Hornbeck PV, Zhang B, Murray B, Kornhauser JM, Latham V, Skrzypek E. PhosphoSitePlus, 2014: mutations, PTMs and recalibrations. Nucleic Acids Res. 2015; 43:D512-20.

44. Ross KE, Arighi CN, Ren J, Huang H, Wu CH. Construction of protein phosphorylation networks by data mining, text mining and ontology integration: analysis of the spindle checkpoint. Database (Oxford). 2013; 2013: bat038.

45. Babu MM, van der Lee R, de Groot NS, Gsponer J. Intrinsically disordered proteins: regulation and disease. Curr Opin Struct Biol. 2011; 21:432-40.

46. Wright PE, Dyson HJ. Intrinsically disordered proteins in cellular signalling and regulation. Nat Rev Mol Cell Biol. 2015; 16:18-29.

47. Lee TI, Young RA. Transcriptional regulation and its misregulation in disease. Cell. 2013; 152:1237-51.
48. Nishi H, Hashimoto K, Panchenko AR. Phosphorylation in Protein-Protein Binding: Effect on Stability and Function. Structure. 2011; 19:1807-15.

49. Nishi H, Fong JH, Chang C, Teichmann SA, Panchenko AR. Regulation of protein-protein binding by coupling between phosphorylation and intrinsic disorder: analysis of human protein complexes. Mol Biosyst. 2013; 9:1620-6.

50. England J, Pang KL, Parnall M, Haig MI, Loughna S. Cardiac troponin $\mathrm{T}$ is necessary for normal development in the embryonic chick heart. J Anat. 2016; 229:436-49.

51. Brody MJ, Cho E, Mysliwiec MR, Kim TG, Carlson CD, Lee KH, Lee Y. Lrrc10 is a novel cardiac-specific target gene of Nkx2-5 and GATA4. J Mol Cell Cardiol. 2013; 62:237-46.

52. Sambrook J, Fritsch E, Maniatis T. Molecular cloning: A laboratory manual. 2nd ed. NY: Cold Spring Habor Press Cold Spring Harbor. 1989.

53. Thompson JD, Gibson T, Higgins DG. Multiple sequence alignment using ClustalW and ClustalX. Curr Protoc Bioinformatics. 2002; Chapter 2:Unit 2.3.

54. Schrödinger Release 2017-2: Desmond Molecular Dynamics System, D. E. Shaw Research, New York, NY, 2017. Maestro-Desmond Interoperability Tools, Schrödinger, New York, NY, 2017.

55. Price DJ, Brooks CL 3rd. A modified TIP3P water potential for simulation with Ewald summation. J Chem Phys. 2004; 121:10096-103.

56. Kant S, Asthana S, Missiakas D, Pancholi V. A novel STK1-targeted small-molecule as an "antibiotic resistance breaker" against multidrug-resistant Staphylococcus aureus. Sci Rep. 2017; 7:5067.

57. Evans DJ, Holian BL. The nose-hoover thermostat. J Chem Phys. 1985; 83:4069-74.

58. Martyna GJ. Remarks on "Constant-temperature molecular dynamics with momentum conservation". Phys Rev E Stat Phys Plasmas Fluids Relat Interdiscip Topics. 1994; 50:3234-36.

59. Darden T, Perera L, Li L, Pedersen L. New tricks for modelers from the crystallography toolkit: the particle mesh Ewald algorithm and its use in nucleic acid simulations. Structure. 1999; 7:R55-R60. 